\title{
Transcritical riddling in a system of coupled maps
}

\author{
Popovych, O.; Maistrenko, Yu.; Mosekilde, Erik; Pikovsky, A.S.; Kurths, J.
}

Published in:

Physical Review E. Statistical, Nonlinear, and Soft Matter Physics

Link to article, DOI:

10.1103/PhysRevE.63.036201

Publication date:

2001

Document Version

Publisher's PDF, also known as Version of record

Link back to DTU Orbit

Citation (APA):

Popovych, O., Maistrenko, Y., Mosekilde, E., Pikovsky, A. S., \& Kurths, J. (2001). Transcritical riddling in a system of coupled maps. Physical Review E. Statistical, Nonlinear, and Soft Matter Physics, 63(3), 036201. https://doi.org/10.1103/PhysRevE.63.036201

\section{General rights}

Copyright and moral rights for the publications made accessible in the public portal are retained by the authors and/or other copyright owners and it is a condition of accessing publications that users recognise and abide by the legal requirements associated with these rights.

- Users may download and print one copy of any publication from the public portal for the purpose of private study or research.

- You may not further distribute the material or use it for any profit-making activity or commercial gain

- You may freely distribute the URL identifying the publication in the public portal 


\title{
Transcritical riddling in a system of coupled maps
}

\author{
O. Popovych, ${ }^{1}$ Yu. Maistrenko, ${ }_{1}^{1}$ E. Mosekilde, ${ }^{2}$ A. Pikovsky, ${ }^{3}$ and J. Kurths ${ }^{3}$ \\ ${ }^{1}$ Institute of Mathematics, National Academy of Sciences of Ukraine, 01601 Kiev, Ukraine \\ ${ }^{2}$ Department of Physics, Technical University of Denmark, DK-2800 Lyngby, Denmark \\ ${ }^{3}$ Department of Physics, Potsdam University, PF 601553, 14415 Potsdam, Germany
}

(Received 23 May 2000; published 14 February 2001)

\begin{abstract}
The transition from fully synchronized behavior to two-cluster dynamics is investigated for a system of $N$ globally coupled chaotic oscillators by means of a model of two coupled logistic maps. An uneven distribution of oscillators between the two clusters causes an asymmetry to arise in the coupling of the model system. While the transverse period-doubling bifurcation remains essentially unaffected by this asymmetry, the transverse pitchfork bifurcation is turned into a saddle-node bifurcation followed by a transcritical riddling bifurcation in which a periodic orbit embedded in the synchronized chaotic state loses its transverse stability. We show that the transcritical riddling transition is always hard. For this, we study the sequence of bifurcations that the asynchronous point cycles produced in the saddle-node bifurcation undergo, and show how the manifolds of these cycles control the magnitude of asynchronous bursts. In the case where the system involves two subpopulations of oscillators with a small mismatch of the parameters, the transcritical riddling will be replaced by two subsequent saddle-node bifurcations, or the saddle cycle involved in the transverse destabilization of the synchronized chaotic state may smoothly shift away from the synchronization manifold. In this way, the transcritical riddling bifurcation is substituted by a symmetry-breaking bifurcation, which is accompanied by the destruction of a thin invariant region around the symmetrical chaotic state.
\end{abstract}

DOI: 10.1103/PhysRevE.63.036201

PACS number(s): 05.45.Xt, 05.45.Ac

\section{INTRODUCTION}

\section{A. Ensembles of coupled chaotic oscillators}

In physics, biology, and other fields of science one often encounters systems in which a large assembly of oscillators through their mutual interaction produce different forms of collective behavior at the macroscopic level [1-3]. Examples of this type of system may most easily be found in the living world, e.g., the phase-locking of chirps of neighboring males of certain species of grasshoppers [4], the widespread synchronous calcium oscillations associated with the bursting electrical activity of pancreatic $\beta$ cells [5], or the recently reported sustained glycolytic oscillations in suspensions of yeast cells [6]. However, similar phenomena also arise in the study of Josephson junction arrays [7], in multimode laser systems [8], and in charge density waves [9].

In general, the individual oscillators of the ensemble will not be identical, but their parameters will be distributed over certain intervals. Similarly, the interaction network may also have an inhomogeneous structure with combinations of local and global coupling mechanisms and with more or less random variations in the individual coupling strengths. In view of obtaining a better understanding of problems of this nature, a significant number of investigators have studied the properties of large populations of self-sustained oscillators [10-12]. In particular, it has been shown [13] that the onset of mutual entrainment, which occurs when the interaction exceeds a certain threshold, bears certain analogies with a second-order phase transition.

However, under far-from-equilibrium conditions, besides self-sustained oscillations, each element in the ensemble may exhibit complicated bifurcation scenarios leading to deterministic chaos and to a variety of coexisting solutions. A preliminary study of coupled chaotic oscillators was per- formed by Bohr et al. [14] who simulated 256 interacting nephrons on a parallel computer. More recently, Pikovsky et al. [15] have performed a numerical study of synchronization phenomena in a population of 3000-5000 globally coupled Rössler oscillators. They demonstrated that the emergence of coherent behavior (and, hence, of a nonvanishing macroscopic mean field) is related to a synchronization of the phases of the individual oscillators whereas the amplitudes continue to behave quite differently and are chaotic [16]. With further increase of the coupling parameter, amplitude synchronization of the interacting oscillators can occur, and a large number of coexisting clustered states may be observed. This was shown by Zanette and Mikhailov [17] for a system of 1000 globally coupled Rössler oscillators. At a first sight, the assumed global (or all-to-all) coupling may appear a little unusual. However, it represents a realistic coupling type, in particular, in many biological systems where the cells (or functional units) are stimulated by signals that are controlled by the total activity of these elements.

To fully account for the high-dimensional dynamics of a large population of interacting chaotic oscillators is beyond the range of present understanding. Such systems will typically exhibit an extremely large number of coexisting limiting states, each characterized by its own basin of attraction. Some of the states may be point cycles or quasiperiodic attractors (tori), and others may represent various forms of synchronous chaotic states. Among these there may be Milnor attractors [18] for which the basins are riddled with initial conditions leading to other limiting states [19].

\section{B. Clustering in globally coupled maps}

As a simplified approach to the above problem, Kaneko [20] has considered a symmetrical system of $N$ globally coupled one-dimensional maps (oscillators) 


$$
x_{i}(n+1)=(1-\varepsilon) f\left(x_{i}(n)\right)+\frac{\varepsilon}{N} \sum_{j=1}^{N} f\left(x_{j}(n)\right),
$$

where $i=1, \ldots, N$ is a space index for the $N$-dimensional state vector $\mathbf{x}(n)=\left\{x_{i}(n)\right\}_{i=1}^{N} . \quad \varepsilon \in \mathbb{R}$ is the coupling parameter, and $f: \mathbb{R} \rightarrow \mathbb{R}$ is a one-dimensional nonlinear map that can generate chaotic dynamics for the individual oscillator in the absence of coupling. The simplest form of asymptotic dynamics that can occur in system (1) is the fully synchronized (or coherent) behavior in which all elements display the same temporal variation. In this case the motion is restricted to a one-dimensional invariant manifold, the main diagonal in phase space.

For certain values of the coupling parameter, the state of full synchronization may attract all or almost all initial conditions. For other values of $\varepsilon$, different types of clustering are observed [20], i.e., the population of oscillators splits into groups with different dynamics, but such that all oscillators within a given group asymptotically move in synchrony. Two-cluster behavior, for instance, is characterized by the dominance of dynamics for which

$$
\begin{gathered}
x_{i_{1}}=x_{i_{2}}=\cdots=x_{i_{N_{1}}} \stackrel{\text { def }}{=} x, \\
x_{i_{N_{1}+1}}=x_{i_{N_{1}+2}}=\cdots=x_{i_{N}}=y,
\end{gathered}
$$

with $N_{1}$ and $N_{2}=N-N_{1}$ denoting the number of synchronized elements in each of the two clusters. This is usually the first type of clustering to occur as the coupling parameter $\varepsilon$ is reduced, and the state of full synchronization breaks down.

Under the conditions (2), the coupled map system (1) transforms precisely into a system of two coupled onedimensional maps of the form

$$
\begin{aligned}
{\left[\begin{array}{l}
x(n+1) \\
y(n+1)
\end{array}\right] } & =F\left[\begin{array}{l}
x(n) \\
y(n)
\end{array}\right] \\
& =\left[\begin{array}{c}
f(x(n))+p \varepsilon[f(y(n))-f(x(n))] \\
f(y(n))+(1-p) \varepsilon[f(x(n))-f(y(n))]
\end{array}\right] .
\end{aligned}
$$

Hence, we can study the breakdown of full synchronization and the appearance of two-cluster dynamics by means of system (3) with parameter $p$ describing the distribution of oscillators between the two clusters. (More precisely, $p$ $=N_{2} / N$ denotes the fraction of the total population that synchronizes into state $y$.) For $N=3$, for example, with two clusters $x_{1}=x_{2} \stackrel{d e f}{=} x$ and $x_{3} \stackrel{d e f}{=} y$, the dynamics of Eq. (1) is described by Eq. (3) with $p=1 / 3$. Clearly, for $N=3$, twocluster dynamics can be realized in $3 ! /(2 ! 1 !)=3$ different ways. Hence, we have three two-cluster states depending on the index of subspaces. For larger values of $N$, the possible realizations of a given cluster distribution grow very rapidly.

System (3) describes the dynamics of any of the $N ! /\left[N_{1} !\left(N-N_{1}\right) !\right]$ two-cluster states defined by Eq. (2).
However, it says nothing about the stability of these states in the full $N$-dimensional phase space, i.e., about their possible break-up into three- or higher-dimensional cluster states. Indeed, any of the two-cluster states may be unstable in the $\mathrm{N}$-dimensional phase space if one (or more) of the corresponding transverse Lyapunov exponents is positive. In the present paper we shall not concern ourselves with this problem (which has been the subject of a number of recent investigations [21]). Instead, we shall consider the mechanisms involved in the transition from one- to two-cluster dynamics for the whole range of possible distributions of the oscillators between the two clusters. We shall also provide a detailed overview of the types of two-cluster dynamics that can occur as the parameters $\varepsilon$ and $p$ are varied.

\section{Symmetrically coupled maps}

In the case of symmetrical linear coupling

$$
\begin{aligned}
& x(n+1)=f(x(n))+\varepsilon[y(n)-x(n)], \\
& y(n+1)=f(y(n))+\varepsilon[x(n)-y(n)],
\end{aligned}
$$

we have previously described the processes by which chaotic synchronization is lost when the one-dimensional map $f$ is given by the logistic map $f(x)=a x(1-x)[22,23]$. We have also presented different scenarios for the local and global bifurcations that take place after the first transverse destabilization of a periodic orbit embedded in the synchronized chaotic state. In this connection the role of absorbing areas was emphasized in restraining the dynamics of the coupled map system, once the chaotic synchronization breaks down [24]. The notion of an absorbing area derives from the theory of two-dimensional noninvertible maps [25] and refers to an attracting invariant (or semi-invariant) region of phase space that absorbs all or almost all trajectories from a neighborhood in a finite number of iterations, and from which trajectories can never escape. Absorbing areas are bounded by segments of critical curves that are obtained as successive images of the curves in phase space where the Jacobian of the two-dimensional, noninvertible map vanishes. Moreover, parts of the boundary may be made up by unstable manifolds of saddle cycles. Recently, Ashwin and Terry [26] proposed a potential extension of the concept of absorbing areas, termed absorbing regions, and discussed their relations to weak and Milnor attractors.

The case of two symmetrically coupled logistic maps with nonlinear coupling has been considered by Astakhov et al. [27]. They have followed some of the bifurcations that take place after the first transverse destabilization of a lowperiodic synchronous saddle cycle in the diagonal $D$ $=\{(x, y) \mid x=y\}$. A subsequent paper [28] studied the influence of a parameter mismatch on the desynchronization transitions in the same system.

\section{Outline of the paper}

As described above, the purpose of the present paper is to study the transition from fully synchronized to two-cluster dynamics in a system given by Eq. (3) for an arbitrary dis- 
tribution of the sites between the two clusters, i.e., for arbitrary values of the asymmetry parameter $p \in[0,1]$. For symmetrically coupled, identical maps $\left(p=\frac{1}{2}\right)$, the first transverse destabilization of a saddle cycle embedded in the synchronized chaotic state (and, hence, the transition to riddling) occurs via a transverse period-doubling bifurcation or via a transverse pitchfork bifurcation. The presence of asymmetry in the coupling $\left(p \neq \frac{1}{2}\right)$ does not change the general form of the transverse period-doubling bifurcation. The transverse pitchfork bifurcation, on the other hand, is transformed into a saddle-node bifurcation leading to the formation of a couple of point cycles off the synchronization manifold. The riddling bifurcation hereafter becomes transcritical, i.e., it involves the exchange of stability between one of the just-appeared point cycles and the saddle cycle on the diagonal.

We study the sequence of bifurcations that the asynchronous point cycles produced in the saddle-node bifurcation undergo as the coupling strength $\varepsilon$ and the asymmetry parameter $p$ are varied. We show how the unstable manifolds of one or both of these cycles control the global dynamics of the system after riddling has occurred and, hence, the character of the riddling bifurcation. The transcritical riddling bifurcation is found to always be hard.

In the case where there is a parameter mismatch so that two slightly different maps $f_{a_{1}}(x)$ or $f_{a_{2}}(x)$ are associated with the individual space points $x_{i}, i=1, \ldots, N$, the transcritical riddling bifurcation is replaced by two saddle-node bifurcations. Alternatively, if the sign of the parameter mismatch is different (in relation to the sizes of the two subpopulations), the saddle cycle involved in the transverse destabilization of the synchronized chaotic state smoothly shifts away from the synchronization manifold. Again we show that the stable and unstable manifolds of the asynchronous point cycles play an essential role for the dynamics of the system. In this way, the transcritical riddling bifurcation is replaced by a symmetry-breaking bifurcation that destroys the thin invariant region existing around the nearly symmetric chaotic state.

The paper concludes with a short description of the bifurcations of the attractors that are placed away from the diagonal and, therefore, are responsible for the dynamics in the two-cluster states of the original $\mathrm{N}$-dimensional system.

\section{THE RIDDLING BIFURCATION}

Consider the two-dimensional coupled map system (3) with $f(x)$ defined as the one-dimensional logistic map $f_{a}(x)=a x(1-x)$. Let the parameter $a \in(3,4)$ be chosen such that the map $f: x \mapsto f_{a}(x)$ has a chaotic attractor $I \subset[0,1]$. Transverse to the diagonal $D=\{(x, y) \mid x=y\}$ the eigenvalue of the map $F(3)$ is equal to $\nu_{\perp}=f_{a}^{\prime}(x)(1-\varepsilon)$. So, for $\varepsilon=1$ we have $\nu_{\perp}=0$, and the diagonal $D$ is superstable. Moreover, at $\varepsilon=1$ any initial point $(x(0), y(0))$ $\in \mathbb{R}^{2}$ is mapped onto $D$ in a single iteration under the action of $F$.

This superstability of the diagonal obviously ensures the existence of an interval for the coupling parameter $\varepsilon$ around $\varepsilon=1$ where the fully synchronous chaotic state $I_{D}$

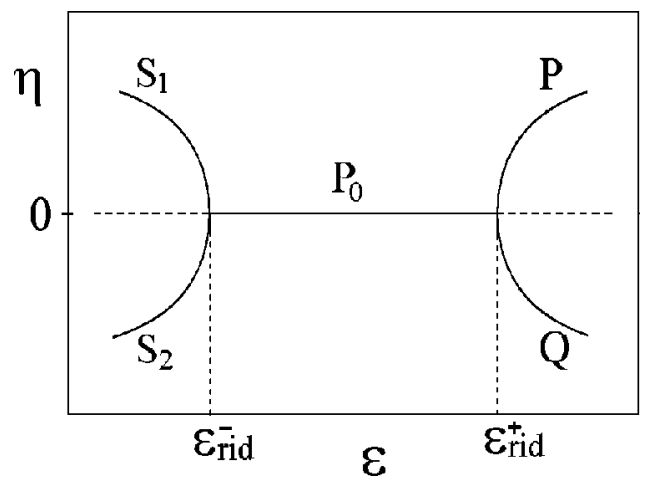

FIG. 1. Transverse bifurcations of the fixed point $P_{0}$ lying in the synchronous state $I_{D}$ for the map (3) in the case of symmetrical coupling $\left(p=\frac{1}{2}\right)$. To the left (at $\varepsilon=\varepsilon_{\text {rid }}^{-}$), a supercritical perioddoubling bifurcation takes place giving rise to a saddle period-2 cycle $\gamma_{2}=\left\{S_{1}, S_{2}\right\}$. To the right (at $\varepsilon=\varepsilon_{\text {rid }}^{+}$), a supercritical pitchfork bifurcation occurs giving rise to two saddle fixed points $P$ and $Q$. The variable $\eta$ is defined as $\eta=(y-x) / 2$. Dashed lines at $\eta$ $=0$ are for the repelling fixed point $P_{0}$.

$=\{(x, y) \mid x=y \in I\}$ is asymptotically stable. When the coupling parameter $\varepsilon$ varies beyond this interval, the synchronous state $I_{D}$ loses its asymptotic stability. In the literature such a loss of stability is referred to as a riddling bifurcation $[29,30]$. As described in a number of papers [22,27,29-32], the riddling bifurcation takes place when some saddle cycle embedded in the synchronous chaotic state loses its stability in the direction transverse to the diagonal. Often the saddle periodic cycle that first loses its transverse stability is of relatively low periodicity [33]. The riddling bifurcation for the map (3) with symmetrical coupling ( $\left.p=\frac{1}{2}\right)$ was studied, e.g., by Astakhov et al. [27]. Below we describe the corresponding bifurcation in the case of nonsymmetrical coupling $\left(p \neq \frac{1}{2}\right)$. As we shall see, it can differ essentially from that of the symmetrical case.

\section{A. Symmetrical coupling}

For $a>a_{0} \cong 3.678$, if the map $f_{a}$ has a one-piece chaotic attractor $I$, the riddling bifurcation (loss of asymptotic stability) of the one-piece synchronous state $I_{D} \subset D$ takes place when the saddle fixed point $P_{0}\left(x^{*}, x^{*}\right)$ embedded in $I_{D}$ loses its stability in the direction transverse to $D$ and becomes a repellor. Here $x^{*}=1-1 / a$ is a fixed point of the logistic map $f_{a}$. It can easily be computed that the bifurcation occurs at $\varepsilon=\varepsilon_{\text {rid }}^{ \pm}=1 \pm 1 /(a-2)$ when the transverse eigenvalue $\nu_{\perp}$ $=(2-a)(1-\varepsilon)$ of the fixed point $P_{0}$ becomes greater than 1 in absolute value.

Consider system (3) with the symmetrical coupling, i.e., for $p=\frac{1}{2}$. A schematic diagram of transverse bifurcations of the fixed point $P_{0}$ is presented in Fig. 1. At $\varepsilon=\varepsilon_{\text {rid }}^{-}$the riddling bifurcation involves a transverse period-doubling bifurcation of the fixed point $P_{0}$ (the transverse eigenvalue of $P_{0}$ leaves the unit circle through -1 ). The bifurcation gives rise to the birth of an antisymmetric period-2 saddle cycle $\gamma_{2}=\left\{S_{1}, S_{2}\right\}$ whose points gradually move away from the diagonal when $\varepsilon$ continues to decrease. At $\varepsilon=\varepsilon_{\text {rid }}^{+}$the riddling bifurcation has the form of a transverse pitchfork bi- 


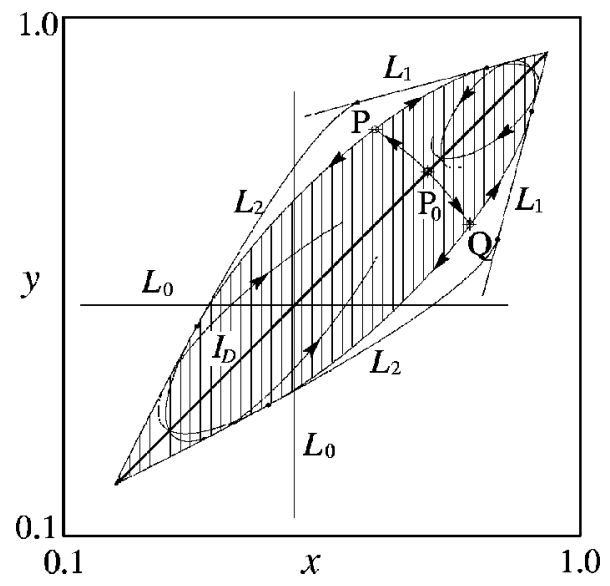

FIG. 2. Absorbing area (crosshatched) for the map $F$ in the symmetric case $\left(p=\frac{1}{2}\right)$ after a supercritical pitchfork bifurcation of the fixed point $P_{0}$. The absorbing area is bounded partly by arcs of the critical curves $L_{1}$ and $L_{2}$ and partly by unstable manifolds of the asynchronous point cycles $P$ and $Q$ born in the pitchfork bifurcation. The parameters are $a=3.8, p=0.5$, and $\varepsilon=1.57$.

furcation (the transverse eigenvalue of $P_{0}$ becomes greater than +1$)$. After the bifurcation, two saddle fixed points $P$ and $Q$ close to the diagonal appear. They again move away from the diagonal when $\varepsilon$ increases.

Both the period-doubling and the pitchfork transverse bifurcations at $\varepsilon=\varepsilon_{\text {rid }}^{ \pm}$are supercritical. It follows that the corresponding riddling bifurcations are soft [23,31], i.e., immediately after the bifurcation there exists an invariant region of infinitesimal transverse size, called the absorbing area $[23,24]$, which envelops all trajectories starting close to the chaotic attractor $I_{D}$ in the diagonal.

An example of the absorbing area that appears after the supercritical pitchfork riddling bifurcation is crosshatched in Fig. 2. As illustrated in this figure, the boundary of the absorbing area is partially composed of unstable manifolds of the antisymmetric saddle fixed points $P$ and $Q$, and partially (near the corner points) by the critical curves $L_{1}$ and $L_{2}$ which are the first and the second iterations by $F$ of the locus of points where the Jacobian of the map $F$ vanishes:

$$
L_{0}=\{(x, y) \mid x=1 / 2 \text { or } y=1 / 2\} .
$$

The absorbing area that appears after the period-doubling bifurcation has a similar shape. The only difference is that now unstable manifolds of the antisymmetric period-2 saddle cycle $\gamma_{2}=\left\{S_{1}, S_{2}\right\}$ take part in creating the boundary of the absorbing area.

Just after the supercritical riddling bifurcation (whether it is caused by a transverse period-doubling or pitchfork bifurcation), the transversal size of the absorbing area is infinitesimal. When moving away from the bifurcation point its width grows $\sim \sqrt{\left|\varepsilon-\varepsilon_{\text {rid }}^{ \pm}\right|}$. This width determines the amplitude of the maximal possible bursts away from the synchronized state.

\section{B. Asymmetric coupling}

The bifurcation points $\varepsilon=\varepsilon_{\text {rid }}^{ \pm}$of the riddling bifurcations are independent on $p$. Indeed, as noted in [32], the transver-

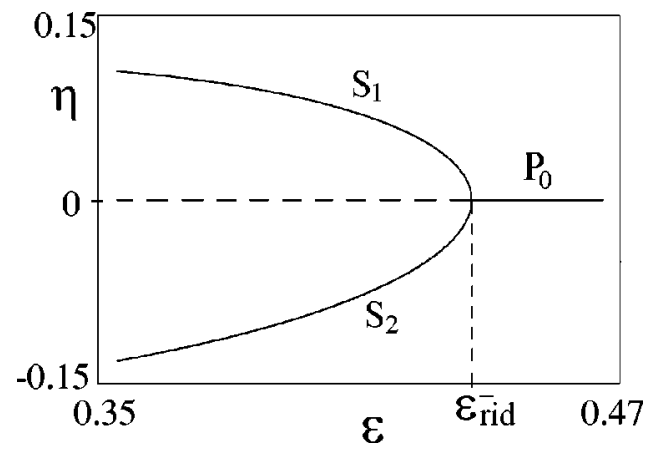

FIG. 3. Diagram of the riddling bifurcation when the fixed point $P_{0} \in I_{D}$ loses its transversal stability through a supercritical perioddoubling bifurcation at $\varepsilon=\varepsilon_{\text {rid }}^{-}$giving rise to a saddle period-2 cycle $\gamma_{2}=\left\{S_{1}, S_{2}\right\}$. Here $\eta=(y-x) / 2$. The dashed line at $\eta=0$ corresponds to a repelling fixed point $P_{0}$. The parameter $p=0.33$.

sal eigenvalue of any symmetrical point cycle only depends on the sum of coupling parameters that for the system (3) equals $d=\varepsilon p+\varepsilon(1-p)=\varepsilon$. Therefore, the parameter points $\varepsilon=\varepsilon_{\text {rid }}^{ \pm}$of the riddling bifurcations are the same for any $p$ $\in[0,1]$.

It is well known $[34,35]$ that period-doubling is a generic bifurcation. Hence, the riddling bifurcation at $\varepsilon=\varepsilon_{\text {rid }}^{-}$persists under perturbations of the system, and it maintains the form of a period-doubling even if the symmetry is broken $\left(p \neq \frac{1}{2}\right)$. As illustrated in Fig. 3, an asymmetric coupling only causes a small difference between the deviations $\eta=(y$ $-x) / 2$ from the diagonal of the points $S_{1}$ and $S_{2}$ for the period-2 saddle cycle $\gamma_{2}=\left\{S_{1}, S_{2}\right\}$.

Let us hereafter focus on how the riddling bifurcation caused by the supercritical pitchfork at $\varepsilon=\varepsilon_{\text {rid }}^{+}$changes with the introduction of an asymmetry in the coupling, i.e., for $p \neq \frac{1}{2}$. To investigate the changes of the transverse pitchfork bifurcation we use a method recently proposed by Maistrenko et al. [23].

Using the linear variable transformation

$$
\xi=(y+x) / 2, \quad \eta=(y-x) / 2
$$

to Eq. (3), we can rewrite the map $F$ in the new variables $\xi$, $\eta:$

$$
\widetilde{F}:\left[\begin{array}{c}
\xi \\
\eta
\end{array}\right] \mapsto\left[\begin{array}{c}
f(\xi)+f^{\prime}(\xi)(2 p-1) \varepsilon \eta-a \eta^{2} \\
f^{\prime}(\xi)(1-\varepsilon) \eta
\end{array}\right],
$$

where $f(\xi)=a \xi(1-\xi)$ is the logistic map and $f^{\prime}(\xi)=a(1$ $-2 \xi)$ its derivative. The variable change (6) is a simple $\pi / 4$ rotation of phase space such that the diagonal $x=y$ for the original map $F$ corresponds to the axis $\eta=0$ in the map $\widetilde{F}$ (plus a scaling with the factor $\sqrt{2}$ ).

For the map $\widetilde{F}$ the riddling bifurcation at $\varepsilon=\varepsilon_{\text {rid }}^{+}$takes place when the transverse eigenvalue $\nu_{\perp}=f^{\prime}\left(\xi^{*}\right)(1-\varepsilon)$ of the fixed point $\widetilde{P}_{0}\left(\xi^{*}, 0\right)$ passes through +1 , where $\xi^{*}$ $=x^{*}=1-1 / a$. For the considered values of parameter $a$ $\in(3,4)$ the fixed point $\xi^{*}$ is unstable for the logistic map $f_{a}$. 
Therefore, the absolute value of the longitudinal eigenvalue $\nu_{\|}=f^{\prime}\left(x^{*}\right)$ of the fixed point $\widetilde{P}_{0}$ is always greater than 1.

Let the coupling parameter be such that the fixed point $\widetilde{P}_{0}$ is a transversely attracting saddle, i.e., $\left|\nu_{\perp}\right|<1$ and the synchronous chaotic state is still before the riddling bifurcation. Then, there exists an invariant one-dimensional transversal manifold $W_{\perp}=\{(\xi, \eta) \mid \xi=\varphi(\eta)\}$ passing through $\widetilde{P}_{0}$. The Taylor series expansion of $\varphi(\cdot)$ in the vicinity of zero is

$$
\varphi(\eta)=\xi^{*}+(1-2 p) \eta+\frac{4 a p(1-p)}{\nu_{\|}-\nu_{\perp}^{2}} \eta^{2}+\cdots,
$$

where dots denote terms of higher order. From the expansion above it follows that the manifold $W_{\perp}$ in the vicinity of the fixed point $\widetilde{P}_{0}$ is smooth up to $C^{2}$ provided that the nonresonant conditions $\nu_{\|} \neq \nu_{\perp}$ and $\nu_{\|} \neq \nu_{\perp}^{2}$ are fulfilled.

Having calculated asymptotically the invariant transverse manifold (8) we can study the action of the two-dimensional map (7) along it. In this way we obtain a one-dimensional map $h: \eta \mapsto h(\eta)$ that is a restriction of $\widetilde{F}$ onto $W_{\perp}$ in the vicinity of zero. The expansion of the map $h$ near zero up to the order 3 has the following form:

$$
\begin{aligned}
h(\eta)= & \nu_{\perp} \eta+2 a(2 p-1)(1-\varepsilon) \eta^{2} \\
& +\frac{8 a^{2} p(1-p)(1-\varepsilon)}{\nu_{\perp}^{2}-\nu_{\|}} \eta^{3}+\cdots
\end{aligned}
$$

The nonresonant conditions $\nu_{\|} \neq \nu_{\perp}$ and $\nu_{\|} \neq \nu_{\perp}^{2}$ guarantee $C^{3}$-smoothness of the map $h(\cdot)$ near zero.

Bifurcation of the fixed point $\eta_{0}=0$ for the onedimensional map $h$ corresponds to the transverse destabilization of the fixed point $P_{0}$ for the original two-dimensional map $F$ given by Eq. (3).

Let $p=\frac{1}{2}$, i.e., the coupling is symmetrical. Then the quadratic term in Eq. (9) vanishes. It immediately follows that before the bifurcation, at $\varepsilon<\varepsilon_{\text {rid }}^{+}$, the map $h$ has a single stable fixed point $\eta_{0}=0$, whereas after the bifurcation, at $\varepsilon$ $>\varepsilon_{\text {rid }}^{+}$, the fixed point $\eta_{0}$ is unstable and in its neighborhood there appear two new nontrivial fixed points that are stable. Therefore, with symmetrical coupling the riddling bifurcation has the form of a supercritical pitchfork bifurcation as shown in Fig. 1.

When the symmetry of the map (3) is broken, the bifurcation diagram undergoes an essential change. Indeed, it can be shown from Eq. (9) that for $p \neq \frac{1}{2}$, and for appropriate values of coupling parameter $\varepsilon<\varepsilon_{\text {rid }}^{+}$, the one-dimensional map $h$ acquires two additional nontrivial fixed points. Both of them are positive for $p<\frac{1}{2}$ and negative for $p>\frac{1}{2}$. One of the nontrivial fixed points being unstable approaches $\eta_{0}=0$ as $\varepsilon \rightarrow \varepsilon_{\text {rid }}^{+}$and passes through the fixed point $\eta_{0}$ at $\varepsilon$ $=\varepsilon_{\text {rid }}^{+}$in a transcritical bifurcation. In this bifurcation the interacting fixed points exchange their stability.

For the original two-dimensional map (3) the riddling bifurcation obeys the following scenario, see Fig. 4. When increasing the coupling parameter $\varepsilon$ beyond 1 , a saddle-node bifurcation occurs at some value of the parameter $\varepsilon=\varepsilon_{s n}$,

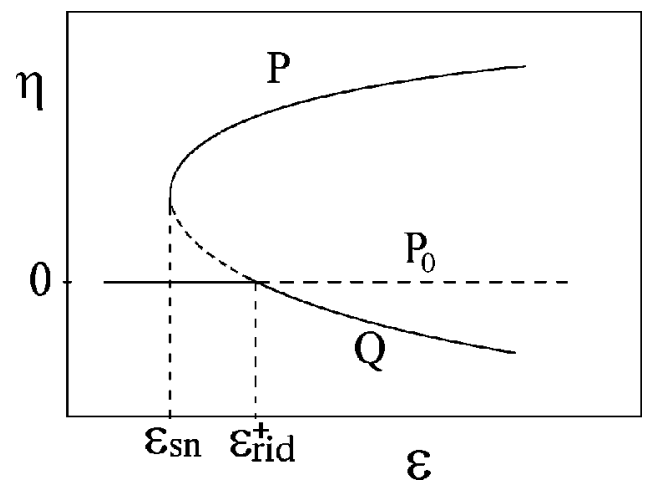

FIG. 4. Diagram of the transcritical riddling bifurcation when the fixed point $P_{0}\left(x^{*}, x^{*}\right) \in I_{D}$ loses its transverse stability through the exchange of stability with another solution. The parameter $p$ $<\frac{1}{2}$ is fixed. For $p>\frac{1}{2}$ the bifurcation diagram changes symmetrically with respect to the line $\eta=0$. Dashed curves correspond to repelling fixed points, and solid curves to saddle points.

producing two fixed points $P$ and $Q$. Both of these fixed points are situated above the diagonal $D$ if $p<\frac{1}{2}$, and under if $p>\frac{1}{2}$. With further increase of $\varepsilon$, the repelling fixed point $Q$ approaches the diagonal $D$. At $\varepsilon=\varepsilon_{\text {rid }}^{+}$it passes through the saddle fixed point $P_{0} \in D$. The fixed points $P_{0}$ and $Q$ exchange their stability. After the transcritical riddling bifurcation, the symmetrical fixed point $P_{0}$ on the diagonal becomes a repellor, and the fixed point $Q$ becomes a saddle lying below (for $p<\frac{1}{2}$ ) and above (for $p>\frac{1}{2}$ ) the diagonal $D$. The bifurcation diagram for the transcritical riddling is shown in Fig. 4.

In the variables $(\xi, \eta)$ [defined in Eq. (6)], the coordinates of the fixed points $P\left(\xi^{+}, \eta^{+}\right)$and $Q\left(\xi^{-}, \eta^{-}\right)$can be expressed as

$$
\xi^{ \pm}=\frac{1}{2}+\frac{1}{2 a(\varepsilon-1)}
$$

and

$$
\eta^{ \pm}=\frac{(1-2 p) \varepsilon \pm \sqrt{(a-1)^{2}(\varepsilon-1)^{2}-4 \varepsilon^{2} p(1-p)}}{2 a(\varepsilon-1)} .
$$

The fixed points $P$ and $Q$ exist for $\varepsilon \geqslant \varepsilon_{s n}$, where

$$
\varepsilon_{s n}=\frac{(a-1)^{2}+2(a-1) \sqrt{p(1-p)}}{(a-1)^{2}-4 p(1-p)}
$$

is the parameter value of the saddle-node bifurcation of $F$.

\section{GLOBAL DYNAMICS AFTER A TRANSCRITICAL RIDDLING}

In this section we shall show that the riddling bifurcation in the system (3), if caused by the transverse transcritical bifurcation of $P_{0}$, is always hard. This implies that immediately after the bifurcation there will be a path for the trajectories to go far away from the attractor $I_{D} \subset D$, even if they start in a very thin neighborhood $U$ of $I_{D}$. In other words, 
any slight coupling asymmetry transforms the soft riddling bifurcation caused by a supercritical pitchfork bifurcation (at $p=\frac{1}{2}$ ) into a hard riddling transition caused by the transcritical bifurcation (at $p \neq \frac{1}{2}$ ). We presume that this type of hard bifurcation will be typical for coupled identical oscillators as soon as the coupling is nonsymmetrical.

\section{A. Absorbing areas}

To throw light on the properties of the transcritical riddling bifurcation, the global dynamics of the noninvertible map $F$ given by the system (3) has to be examined. In this connection, the role of absorbing areas has to be taken into account [24].

Absorbing areas play a fundamental role in the theory of two-dimensional noninvertible maps [25] due to the following strong attracting property: they absorb all or almost all trajectories from their neighborhoods and retain these trajectories forever. Absorbing areas do not arise in diffeomorphisms (since there are no points at which $D F=0$ ).

The role of the absorbing area in connection with chaotic synchronization in systems of coupled noninvertible maps was emphasized in a recent paper by Maistrenko et al. [24]. Here, it was shown how the existence of an absorbing area is important for the distinction between locally and globally riddled basins of attraction as well as between subcritical and supercritical blowout bifurcations $[30,36]$. Only in the presence of an absorbing area can one observe the phenomena of on-off intermittency and attractor bubbling.

When $N$ logistic (or, more generally, noninvertible) maps are coupled, it is easy to see that the resulting $N$-dimensional map $F_{N}$ will be noninvertible as well, i.e., there exist critical hypersurfaces such that $D F_{N}=0$. Hence, when analyzing the global dynamics of $F_{N}$ one may expect the existence of "absorbing volumes" $-N$-dimensional invariant regions whose boundaries are formed (completely or partially) by images of the critical hypersurfaces. However, to the best of our knowledge, so far only two-dimensional noninvertible maps have been analyzed on this property. A generalization of the concept of an absorbing area to the case of $N$-dimensional noninvertible maps remains an interesting but challenging problem.

\section{B. Absorbing areas for the transcritical riddling}

Consider the synchronized chaotic state $I_{D}$ after the transcritical riddling but before the blowout bifurcation (sometimes this regime is called the regime of weak synchronization, see, e.g., the paper by Maistrenko et al. in Ref. [21]). The chaotic attractor $I_{D}$ is still attracting in average, i.e., its Lyapunov exponent $\lambda_{\perp}$ responsible for the growth of transverse perturbations is negative (see Sec. IV for details). For example, if $a=3.8$ the riddling bifurcation takes place at $\varepsilon$ $\approx 1.55$, and the synchronous state $I_{D}$ is attracting in the average up to $\varepsilon \approx 1.65$ where $\lambda_{\perp}$ changes its sign from negative to positive, and the blowout bifurcation takes place.

In the parameter interval $1.55 \leqslant \varepsilon \leqslant 1.65(a=3.8)$, the state $I_{D}$ is a measure-theoretic Milnor attractor: It attracts a positive Lebesgue measure set of points from its neighborhood $[19,30]$. The topological properties of the basin of at-

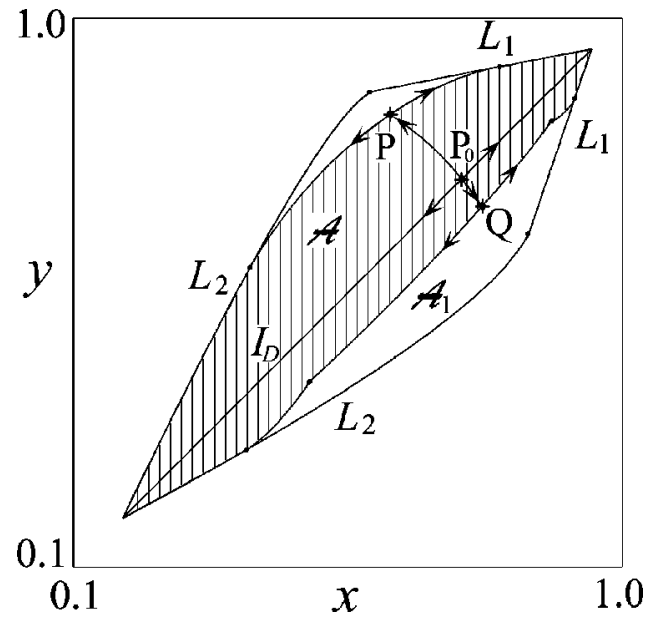

FIG. 5. Absorbing areas $\mathcal{A}$ (crosshatched) and $\mathcal{A}_{1}$ after a transcritical riddling bifurcation. Smaller area $\mathcal{A}$ is bounded by segments of unstable manifolds of saddle fixed points $P$ and $Q$, and by the segments $L_{1}$ and $L_{2}$ of critical curves. Larger absorbing area $\mathcal{A}_{1}$ is bounded only by the critical curves $L_{1}$ and $L_{2}$. The parameters are $a=3.8, \varepsilon=1.57$, and $p=0.45$.

traction are still far from being understood in details. Certain progress has been made in the case of piecewise-linear maps. In particular, Pikovsky and Grassberger [37] have conjectured that for two coupled tent maps, in the regime of weak synchronization, periodic points are dense in the vicinity of the synchronous chaotic state. A proof of this conjecture was recently announced by Glendinning [38].

A state portrait of system (3) after the transcritical riddling bifurcation is presented in Fig. 5. There exists an invariant region $\mathcal{A}$ (crosshatched) around the chaotic attractor $I_{D} \subset D$. As before, this invariant region is referred to as an absorbing area [25]: its boundary is composed partially of segments (arcs) of unstable manifolds of the saddle fixed points $P$ and $Q$, and partially of segments of two consecutive $F$ images of the critical curves $L_{0}$ denoted by $L_{1}$ and $L_{2}$, respectively. For simplicity, we shall refer to $L_{1}$ and $L_{2}$ as critical curves too. Inspection of Fig. 5 also shows how the absorbing area $\mathcal{A}$ is embedded in a larger absorbing area $\mathcal{A}_{1}$ whose boundary is delineated entirely by the critical curves $L_{1}$ and $L_{2}$. In certain parts of the phase space, the boundaries of the two absorbing areas coincide.

Our calculations provide evidence that this type of phase portrait containing two nested absorbing areas is typical for system (3) in the parameter region after the transcritical riddling bifurcation and before stabilization of the fixed point $P$ (see Fig. 7). When a trajectory starts near the chaotic set $I_{D}$, after a number of iterations it will typically fall in a small neighborhood of the fixed point $P_{0} \in I_{D}$ and here it attains a finite probability of going away from the diagonal along the separatrix connecting $P_{0}$ with $P$ (or alternatively $P_{0}$ with $Q)$. The deviation of $P$ from the diagonal is larger than that of $Q$. Hence, the distance to $P$ provides an upper bound to the maximum amplitude in the burst of the trajectories away from the synchronous state. Moreover, at the point of transition the distance $P P_{0}$ is finite, and right after the transition, bursts of finite amplitude can therefore be observed. This is 


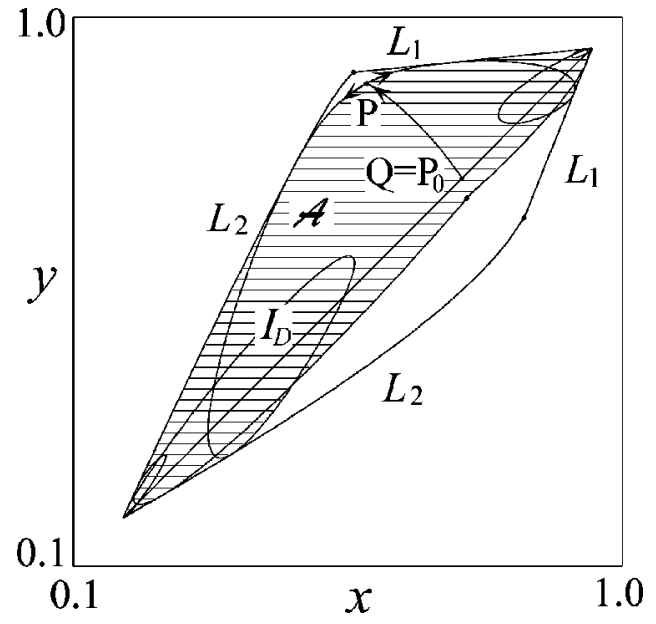

FIG. 6. Absorbing area $\mathcal{A}$ (crosshatched) at the moment of transcritical riddling bifurcation. Parameters are $a=3.8, \varepsilon=1.555$, and $p=0.4$.

in contrast to the symmetrical case, where the amplitude of the bursts grows gradually with the parameter.

\section{Creation and development of the absorbing areas}

Let us follow the evolution of the global dynamics of the map $F$, when the control parameter $\varepsilon$ passes through the bifurcation value $\varepsilon_{\text {rid }}^{+}$of the transcritical riddling (Fig. 4). As we shall see, the sequence of bifurcations observed differs from that of the analogous in the symmetrical case $p=\frac{1}{2}$ reported in [27] and outlined in Sec. II A above.

Before the riddling bifurcation, the attractor $I_{D}$ in the diagonal is asymptotically stable for system (3); it attracts all trajectories from a sufficiently small neighborhood [30]. At the bifurcation moment $\varepsilon=\varepsilon_{\text {rid }}^{+}$, the attractor $I_{D}$ loses its transverse stability (Fig. 6). The smallest invariant region enveloping $I_{D}$ is now an absorbing area $\mathcal{A}$ created by the unstable manifolds of the saddle fixed point $P$ and, partially, by the critical curves $L_{1}$ and $L_{2}$. This is the area of the type described above (see Fig. 5). The maximal distance of its boundary from the diagonal is approximately indicated by the coordinates of the saddle fixed point $P$. Its deviation from the diagonal is

$$
\left|P P_{0}\right|=\sqrt{2} \frac{(1-2 p) \varepsilon+\sqrt{(a-1)^{2}(\varepsilon-1)^{2}-4 \varepsilon^{2} p(1-p)}}{a(\varepsilon-1)} .
$$

The distance from $P_{0}$ to the lower boundary of $\mathcal{A}$ is clearly smaller. Nevertheless, as can be seen in Fig. 6, it is positive beginning from the bifurcation moment $\varepsilon=\varepsilon_{\text {rid }}^{+}$of the transcritical riddling bifurcation considered. This property is due to the fact that the lower boundary of $\mathcal{A}$ is created by the unstable manifolds of saddle $P$.

\section{Hard transition}

Based on the phase portrait in Fig. 6 for the moment of riddling bifurcation at $\varepsilon=\varepsilon_{\text {rid }}^{+}$, we claim that any small neighborhood of the point $P_{0}=Q$ will eventually spread un- der the action of $F$ throughout the whole region $\mathcal{A}$. Indeed, there is a separatrix $\left(P_{0}, P\right)$ connecting $P_{0}$ with $P$. So, a positive measure set of points from the neighborhood will move towards $P$ along $\left(P_{0}, P\right)$. After approaching $P$ at some distance, these trajectories go along the unstable manifolds of $P$ and then fold on the critical curve $L_{1}$ (those moving to the right) or on $L_{2}$ (those moving to the left). Then they cross the diagonal and pass near the right boundary of $\mathcal{A}$ (which is created by the continuation of the unstable manifolds of $P$ ). After this, as computer simulations show, trajectories begin to move between the upper-right and the lower-left cones of $\mathcal{A}$.

While restrained to the absorbing area $\mathcal{A}$, the trajectories (more precisely, the invariant curves created by the trajectories) cross the separatrix $\left(P_{0}, P\right)$. This implies that some points of the trajectories (those near the intersections) can again be involved in the motion along $\left(P_{0}, P\right)$ in the direction towards $P$. And the situation described above will be repeated giving rise to new intersections of stable and unstable manifolds of $P$. Hence, intersections of stable and unstable manifolds of $P$ are evident from our numerical simulations and lead to a complicated dynamics of the map $F$ inside $\mathcal{A}$. However, we have not looked in detail for possible homoclinic tangencies or considered their persistence. It would be interesting to clarify this question to see whether Newhouse regions $[39,40]$ exist for the system of two coupled logistic maps.

Thus, the boundary of the absorbing area $\mathcal{A}$ can be approached, with any given precision, by trajectories originated in any small neighborhood of the fixed point $P_{0}$ belonging to the attractor $I_{D}$ in the diagonal. The location of the boundary gives exact limits for the deviations of the trajectories from the diagonal when they enter into asynchronous bursts. Hence, beginning right from the moment of the transcritical riddling bifurcation at $\varepsilon=\varepsilon_{\text {rid }}^{+}$, the amplitude of the asynchronous bursts is of the order $O(1)$. We conclude that the transcritical riddling bifurcation is always hard.

This property differs from the analogous riddling bifurcation in the symmetrical case $p=\frac{1}{2}$ as well as from the riddling bifurcation caused by the transverse period-doubling bifurcation, which is soft or hard depending on the supercritical or subcritical nature of the bifurcation causing it [23].

Let us continue to vary the coupling parameter $\varepsilon$ beyond the bifurcation value $\varepsilon_{\text {rid }}^{+}$to observe further changes in the structure of the absorbing area $\mathcal{A}$. After the bifurcation, the fixed point $Q$ becomes a saddle and moves down away from the diagonal. First, it lies inside $\mathcal{A}$ so that its unstable manifolds do not participate in the boundary of $\mathcal{A}$. Then, $Q$ emerges from the interior of $\mathcal{A}$, and its unstable manifolds begin to contribute to the boundary (Fig. 5). The parts of the boundary of $\mathcal{A}$ that are created by the manifolds of $Q$ move down and away. However, due to asymmetry $(p<1 / 2)$, the distance to the lower boundary of $\mathcal{A}$ from the diagonal remains smaller than the distance to the upper boundary given by the manifolds of $P$.

\section{RIDDLING AND BLOWOUT TRANSITIONS}

In this section we describe in more detail the riddling and blowout transitions for the chaotic synchronous state $I_{D}$, 


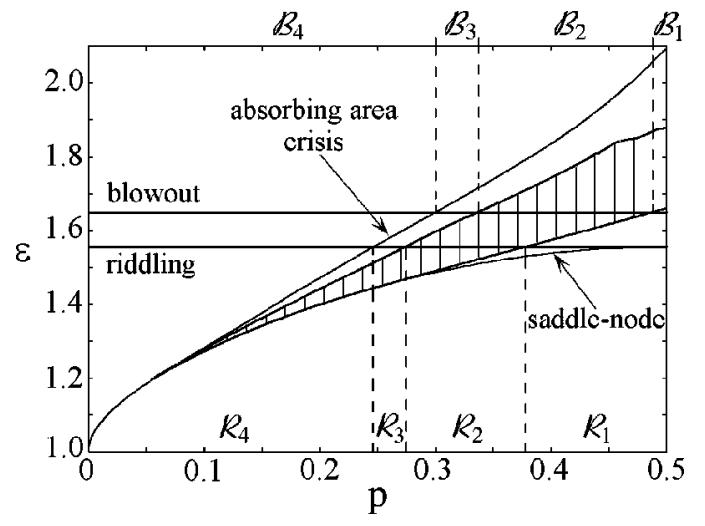

FIG. 7. Bifurcation diagram for the attractor $A_{u p}$ situated above the diagonal. The crosshatched region corresponds to the parameter values where the attractor $A_{u p}$ exists. Riddling $\left(\varepsilon_{\text {rid }}^{+} \approx 1.555 \ldots\right)$ and blowout $\left(\varepsilon_{b l} \approx 1.65 \ldots\right)$ bifurcation lines of the one-piece chaotic attractor $I_{D}$ on the diagonal as well as the curve of saddle-node bifurcation of $P$ and $Q$ (Fig. 4) are also shown. Letters $\mathcal{R}_{1}, \mathcal{R}_{2}$, $\mathcal{R}_{3}, \mathcal{R}_{4}\left(\mathcal{B}_{1}, \mathcal{B}_{2}, \mathcal{B}_{3}, \mathcal{B}_{4}\right)$ denote $p$-intervals (separated by dashed lines) with different types of riddling (blowout) transitions (see text for details). Parameter $a=3.8$.

emphasizing the structure of its basin of attraction. Due to the obvious symmetry of the map (3) with respect to $p=\frac{1}{2}$, we only consider the interval $p \in[0,0.5]$. To be more concrete, let us fix the system parameter $a=3.8$ at that the logistic map $f$ is considered to possess a one-piece chaotic attractor $I$.

An important influence on the riddling and blowout transitions is produced by the presence of another attractor (or infinity) whose basin can come close to the diagonal. In particular, this concerns an attractor $A_{u p}$ (above the diagonal) that may coexist with the stable synchronous state $I_{D}$, and, as a result, may affect the riddling and blowout transitions. The two-parameter $(p, \varepsilon)$ bifurcation diagram of the attractor $A_{u p}$ is presented in Fig. 7. Bifurcations of the attractor $A_{u p}$ are described in more detail in Sec. VI.

The framework of the bifurcation scenario is the following. Let $p$ be fixed and consider what happens as $\varepsilon$ increases starting from 1 . The parameter point $(p, \varepsilon)$ enters into the crosshatched region whose lower boundary, at $\varepsilon=\varepsilon_{s t}$, corresponds to the stabilization of the fixed point $P$ (born in a saddle-node bifurcation together with another fixed point $Q$, see Fig. 4). $P$ becomes a stable node and, with further increase of $\varepsilon$, transforms into an attracting focus. Hereafter, it undergoes a supercritical Hopf bifurcation, and a stable invariant closed curve then appears characterized by quasiperiodic or periodic dynamics on it. This curve is destroyed with further increase of $\varepsilon$, and an attracting chaotic set appears. The upper boundary of the crosshatched region corresponds to the boundary crisis bifurcation, at $\varepsilon=\varepsilon_{c r}$, of the chaotic attractor $A_{u p}$.

The upmost last bifurcation curve in Fig. 7 denoted as "absorbing area crisis" represents the contact bifurcation, at $\varepsilon=\varepsilon_{c o n}$, of the absorbing area $\mathcal{A}$ with the basin of infinity (see $[24,25])$. By destroying the invariant region around the synchronous chaotic state $I_{D}$, this absorbing area crisis results in the appearance of holes belonging to the basin of

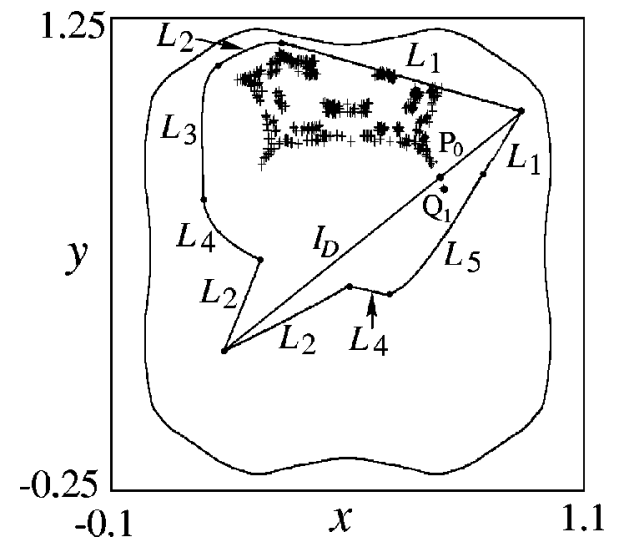

FIG. 8. Synchronous attractor $I_{D}$ and its basin boundary (outer closed curve) after the transcritical riddling bifurcation. By black crosses, a number of periodic orbits belonging to the chaotic saddle $\widetilde{A}_{u p}$ are indicated. Also shown is the boundary of the semi-invariant absorbing area $\mathcal{A}$ that is created by $\operatorname{arcs} L_{k}, k=1, \ldots, 5$ that are the images of critical lines $L_{0}$ from (5). Parameters are $a=3.8, p$ $=0.27$, and $\varepsilon=1.58$.

infinity within the former basin of $I_{D}$. Note that $\varepsilon_{c o n}$ $>\varepsilon_{c r}$. Hence, as $\varepsilon$ is increased, the boundary crisis appears before the contact bifurcation.

\section{A. Riddling transitions}

As discussed in Sec. II, the riddling bifurcation of the chaotic synchronous state $I_{D}$ is independent of $p$ and takes place at $\varepsilon=\varepsilon_{\text {rid }}^{+}=1+1 /(a-2)$. It is caused by the transverse destabilization of the symmetric fixed point $P_{0}\left(x^{*}, x^{*}\right)$ $\in I_{D}, x^{*}=1-1 / a$. For $p=\frac{1}{2}$ this occurs via a supercritical pitchfork bifurcation (Fig. 1), whereas for $p \in[0,1 / 2)$ the transition is transcritical (Fig. 4).

The riddling bifurcation at $\varepsilon=\varepsilon_{\text {rid }}^{+}$may result in a locally or globally riddled basin of $I_{D}$. This depends on the existence of the absorbing area $\mathcal{A}$ around $I_{D}$, and on the absence or presence of another attractor within $\mathcal{A}$ [24].

Let us first consider the case when the transcritical riddling bifurcation leads to a locally riddled basin for the chaotic synchronous state $I_{D}$. Such a transition takes place when the absorbing area $\mathcal{A}$ still exists (that is, before the contact bifurcation) and there are no other attractors within $\mathcal{A}$. As it can be seen in Fig. 7, for the considered value $a$ $=3.8$, this occurs if $p \in \mathcal{R}_{1} \cup \mathcal{R}_{3}=(0.377 \ldots ; 0.5]$ $\cup(0.245 \ldots ; 0.273 \ldots)$.

The difference between the first and the second $p$-intervals of local riddling is that for $p \in(0.377 \ldots ; 0.5]$ the fixed point $P$ has not yet stabilized at the moment of the riddling bifurcation, i.e., $\varepsilon_{\text {rid }}^{+}<\varepsilon_{s t}$ (Fig. 5). In the second case $p \in(0.245 \ldots ; 0.273 \ldots)$, the attractor $A_{u p}$ has already been destroyed through a boundary crisis bifurcation, but the absorbing area $\mathcal{A}$ still exists, i.e., $\varepsilon_{c r}<\varepsilon_{\text {rid }}^{+}<\varepsilon_{\text {con }}$. In place of $A_{u p}$ a chaotic saddle $\widetilde{A}_{u p}$ embedded in the basin of the synchronous chaotic state still exists (Fig. 8).

According to its definition [30], the basin of the synchronous state $I_{D}$ is locally riddled when arbitrarily close to any point in $I_{D}$, there exists a positive measure set of points that 


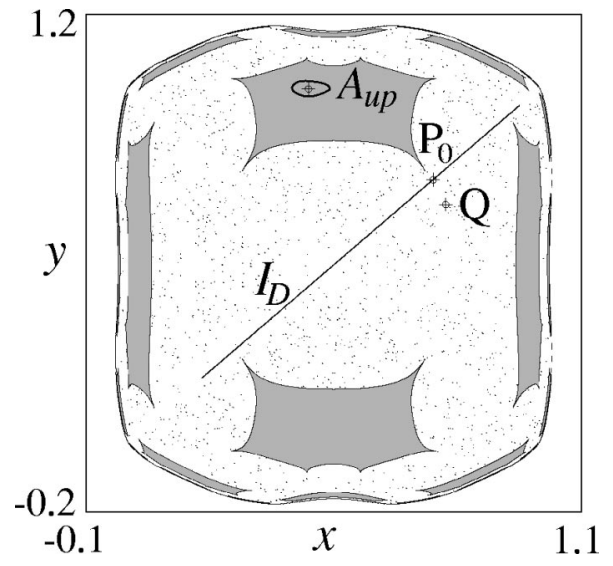

FIG. 9. Transcritical riddling bifurcation may lead to a globally riddled basin of the synchronous state $I_{D}$ with the basin of the attractor $A_{u p}$ above the diagonal. For the given parameters $A_{u p}$ is a closed invariant attracting curve that was born after a supercritical Hopf bifurcation of $P$. The basin of $A_{u p}$ is shown in gray. The basin of $I_{D}$ is densely filled by "dots" that actually are small-diameter regions belonging to the basin of $A_{u p}$. Parameters are $a=3.8, p$ $=0.33$, and $\varepsilon=1.6$.

move away from the diagonal for a finite distance. If there is no other attractor within the invariant absorbing area $\mathcal{A}$, almost all of the trajectories should come back to the synchronous chaotic state. Some of them will be attracted by $I_{D}$, whereas others will repeat the asynchronous bursts. Such a behavior is produced by the transversely repelling fixed point $P_{0} \in I_{D}$ that has an everywhere dense set of preimages in $I_{D}$. Trajectories starting close to such a set will be mapped, first, into a sufficiently small neighborhood of $P_{0}$. Hereafter, they can move away from the diagonal following the separatrix that connects the repelling fixed point $P_{0}$ with the saddle fixed point $P$ [for $p \in(0.377 \ldots ; 0.5]$, see Fig. 5] or with the chaotic saddle $\widetilde{A}_{u p}$ [for $p \in(0.245 \ldots ; 0.273 \ldots)$, see Fig. 8].

If $p \in \mathcal{R}_{2} \cup \mathcal{R}_{4}=(0.273 \ldots ; 0.377) \cup[0 ; 0.245 \ldots)$ the riddling bifurcation leads to a globally riddled basin of the synchronous state $I_{D}$. For $p \in(0.273 \ldots ; 0.377)$ one has $\varepsilon_{s t}<\varepsilon_{\text {rid }}^{+}<\varepsilon_{c r}$, i.e., there exists an attractor $A_{u p}$ above the diagonal in the moment of the bifurcation. The repelling fixed point $Q$, causing the riddling in the transcritical bifurcation with $P_{0} \in I_{D}$, brings with it a stable manifold of the attractor $A_{u p}$ to the diagonal (Fig. 6). Therefore, there exists a tongue of points, with vortex in $P_{0}$, which belong to the basin of attraction of $A_{u p}$. This is illustrated in Fig. 9 where $A_{u p}$ is an invariant closed curve. The fixed point $P_{0}$ has an everywhere dense set of preimages in $I_{D}$ (the map $F$ in $I_{D}$ is noninvertible and mixing). It further follows that the tongue with vortex in $P_{0}$ has preimages in a neighborhood of any point of $I_{D}$. It follows that arbitrarily close to any points of $I_{D}$ there is a positive measure set of points attracted by $A_{u p}$. This is the case when the basin of the synchronous chaotic state $I_{D}$ is globally riddled with the basin of $A_{u p}$.

For $p \in[0 ; 0.245 \ldots)$ an absorbing area contact bifurcation with the basin of infinity takes place before the riddling bifurcation, i.e., $\varepsilon_{c o n}<\varepsilon_{\text {rid }}^{+}$. After the crisis one can observe

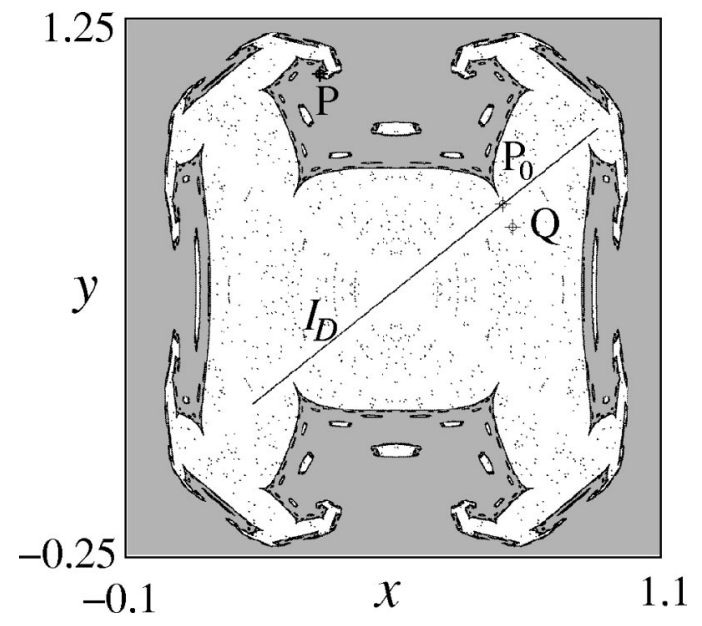

FIG. 10. Globally riddled basin of the synchronous state $I_{D}$ with the basin of infinity after a transcritical riddling bifurcation. Basin of infinity is shown in gray. Parameters are $a=3.8, p=0.245$, and $\varepsilon=1.6$.

"holes to infinity" of a small diameter within the former basin of the synchronous chaotic state $I_{D}$. These holes are accumulated, in particular, in a neighborhood of the fixed point $Q$. Hence, in the moment of the riddling bifurcation, $Q$ brings with it part of the basin of infinity to the fixed point $P_{0} \in I_{D}$. This riddling scenario is similar to what we have discussed for the case when the basin of $I_{D}$ is globally riddled with the basin of $A_{u p}$ (Fig. 9). Therefore, immediately after the riddling bifurcation, the basin of attraction of the synchronous state $I_{D}$ becomes globally riddled with the basin of infinity (Fig. 10).

\section{B. Blowout transition}

The blowout bifurcation [36] of the attracting chaotic synchronous state $I_{D}$ corresponds to its transformation into a chaotic saddle [30]. The bifurcation takes place when the transverse Lyapunov exponent,

$$
\lambda_{\perp}=\lim _{K \rightarrow \infty} \frac{1}{K} \sum_{n=0}^{K-1} \ln \left|f^{\prime}[x(n)](1-\varepsilon)\right|
$$

that is responsible for the average growth of transversal perturbations, changes its sign from negative to positive. The calculation is performed on a typical trajectory $\{(x(n), x(n))\}_{n=0}^{\infty} \subset I_{D}, x(n+1)=f_{a}(x(n))$. For the considered parameter value $a=3.8$, the blowout bifurcation of $I_{D}$ is found to occur at $\varepsilon=\varepsilon_{b l} \approx 1.65$.

The blowout bifurcation for the map (3) may demonstrate different scenarios depending on whether it takes place from a locally or from a globally riddled basin of $I_{D}$. As can be seen in Fig. 7, for $p \in \mathcal{B}_{1} \cup \mathcal{B}_{3}=(0.488 \ldots ; 0.5]$ $\cup(0.3 \ldots ; 0.336 \ldots)$ in the bifurcation moment, the basin of $I_{D}$ is only locally riddled. In this case, the blowout bifurcation is expected to be supercritical, which means that the chaotic attractor $I_{D}$ gradually spreads into the twodimensional phase space [30]. Indeed, as numerical simulations show, trajectories of the new "swelling" attractor $A$ 

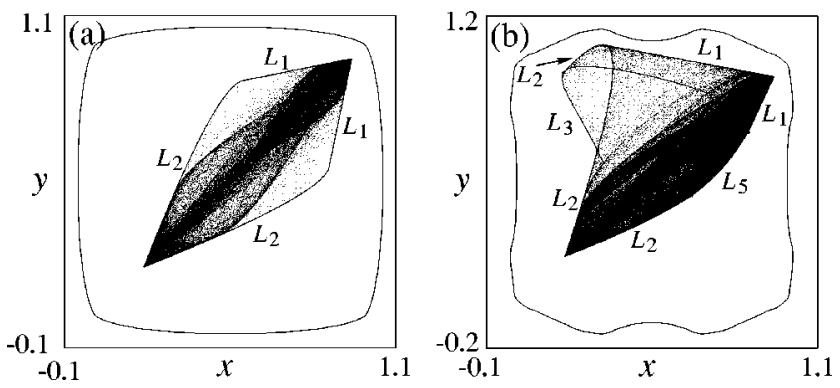

FIG. 11. Attractors for the map (3) and their basin boundaries after a supercritical blowout bifurcations in the presence of a locally riddled basin. Parameters are $a=3.8$, (a) $p=0.498, \varepsilon=1.658$, and (b) $p=0.33, \varepsilon=1.67$.

spend most of the time close to the former attracting synchronous state $I_{D}$, see Fig. 11. This attractor $A$ is bounded by the critical curves $L_{k}$ that are $F^{k}$ iterations of critical curves $L_{0}$ defined by Eq. (5).

If $p \in(0.488 \ldots ; 0.5]$, one has $\varepsilon_{b l}<\varepsilon_{s t}$, i.e., the blowout bifurcation takes place before the stabilization of the fixed point $P$ above the diagonal [Fig. 11(a)]. In the second interval $p \in(0.3 \ldots ; 0.336 \ldots), \varepsilon_{b l}>\varepsilon_{c r}$, and the blowout bifurcation takes place when the chaotic attractor $A_{u p}$ developed from $P$ has already been destroyed [Fig. 11(b)]. Hence, there is a chaotic saddle $\widetilde{A}_{u p}$ in place of it (see also Fig. 8). In this case, the chaotic saddle $\widetilde{A}_{u p}$ may be included in $A$.

The blowout bifurcation is subcritical [30] when it occurs from a globally riddled basin of the synchronous chaotic state $I_{D}$. This is the case for $p \in \mathcal{B}_{2} \cup \mathcal{B}_{4}$ $=(0.336 \ldots ; 0.488 \ldots) \cup[0 ; 0.3 \ldots)$. For the first $p$-interval, in the moment of blowout bifurcation, there exists an attractor $A_{u p}$ over the diagonal and the basin of $I_{D}$ is globally riddled with the basin of $A_{u p}$ (Fig. 9). Hence, above the bifurcation, trajectories are typically attracted by $A_{u p}$. In the second case, $p \in[0 ; 0.3 \ldots)$, the blowout bifurcation takes place when the basin of $I_{D}$ is globally riddled with the basin of infinity (Fig. 10). Above the bifurcation, typical trajectories escape to infinity.

\section{INFLUENCE OF A PARAMETER MISMATCH ON THE RIDDLING BIFURCATION}

In the previous section we described the riddling and blowout transitions when the coupling is asymmetrical ( $p$ $\neq \frac{1}{2}$ ), but the coupled one-dimensional maps are the same. It follows that for any $p \in[0 ; 1]$ the diagonal $D$ remains invariant under the action of the two-dimensional map (3).

Now, to remove the remaining symmetry from the map (3) we add a small parameter mismatch between the coupled maps. For this, consider the system

$$
\begin{gathered}
x(n+1)=f_{a_{1}}(x(n))+p \varepsilon\left[f_{a_{2}}(y(n))-f_{a_{1}}(x(n))\right], \\
y(n+1)=f_{a_{2}}(y(n))+(1-p) \varepsilon\left[f_{a_{1}}(x(n))-f_{a_{2}}(y(n))\right],
\end{gathered}
$$

where, as before, $f_{a}$ denotes the logistic map with parameter $a: f_{a}(x)=a x(1-x)$. Coupling parameters $p$ and $\varepsilon$ are simi-

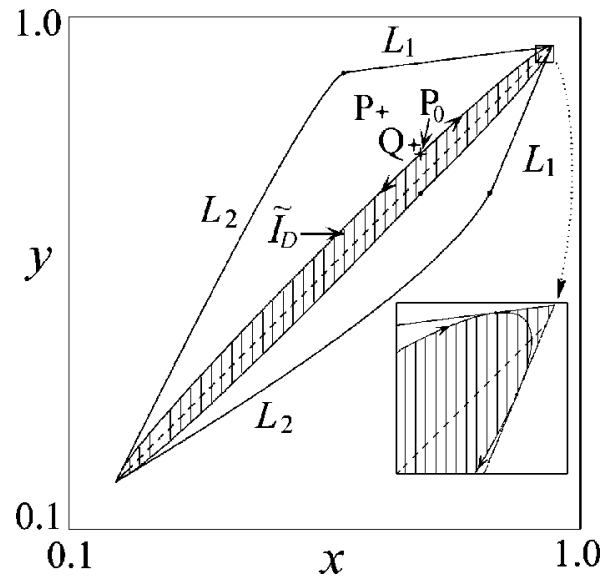

FIG. 12. Absorbing area $\widetilde{I}_{D}$ (crosshatched) that replaces the chaotic synchronous state $I_{D}$ (dashed) when the parameter mismatch $\delta=0.998$ is introduced. Parameters are $a=3.8, \varepsilon=1.538$, and $p$ $=0.4$.

lar to those for the system (3). A mismatch between the one-dimensional maps $f_{a_{1}}$ and $f_{a_{2}}$ can be introduced as follows: $a_{1}=a$ and $a_{2}=a \delta$. For small mismatches the factor $\delta$ has to be close to 1 .

It is evident that for the two-dimensional map $\hat{F}$ given by Eq. (14) the diagonal $D$ is no longer invariant as soon as $\delta$ $\neq 1$. In place of $I_{D} \subset D$ a two-dimensional invariantabsorbing area $\widetilde{I}_{D}$ arises for some range of the coupling parameter $\varepsilon$. Its transverse diameter is small as long as $\delta$ is close to 1 , and vanishes with $\delta \rightarrow 1$. We claim that the transcritical riddling bifurcation is replaced by an interior or an exterior crisis of the absorbing area $\widetilde{I}_{D}$. Moreover, the moment when this happens is shifted with respect to the case without mismatch, and this crisis bifurcation is always hard.

The above phenomenon can be explained by the following changes in the global dynamics of the system (14). There are two distinctive cases depending on the sign of the mismatch, i.e., if $\delta$ is smaller or greater than 1. For $p$ belonging to the interval $\left[0 ; \frac{1}{2}\right)$, the majority of maps are of type $f_{a_{1}}$, and $\delta<1$ implies that most of the maps have the larger nonlinearity parameter $a_{1}$. Conversely, for $\delta>1$, the larger subpopulation of maps have the smaller nonlinearity parameter.

First we fix $\delta=0.998$. When the original system (3) is far enough below the riddling bifurcation, the mismatch produces a shift of the fixed point $P_{0}$ by a small distance above the diagonal. $P_{0}$ is still a saddle, and its unstable manifolds define an invariant absorbing area $\widetilde{I}_{D}$ of a fairly small transverse diameter (crosshatched region in Fig. 12). The boundary of $\widetilde{I}_{D}$ is of the same type as that of $\mathcal{A}$ described in Sec. III, i.e., it consists partially of the unstable manifold of $P_{0}$ and partially of arcs of critical curves $L_{1}$ and $L_{2}$ (see zoom in Fig. 12).

With increasing $\varepsilon$, a saddle-node bifurcation occurs that gives birth to two fixed points $P$ and $Q$ over the region $\widetilde{I}_{D}$ in a way similar to its description in Sec. II above. The repelling node $Q$ moves towards $\widetilde{I}_{D}$, and, at some moment $\varepsilon$ $=\varepsilon_{s n}^{\prime}$, two fixed points $Q$ and $P_{0}$ meet and annihilate one 


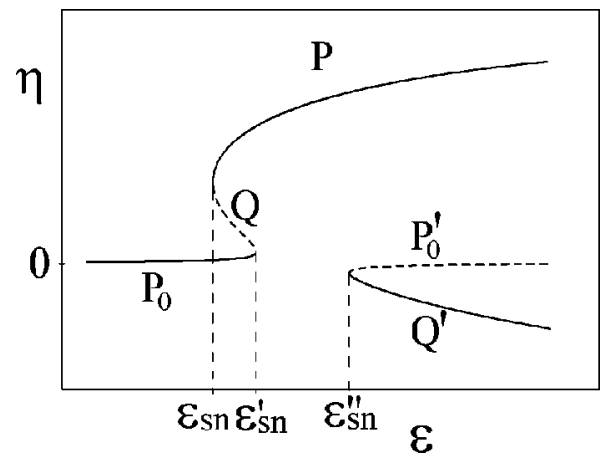

FIG. 13. Diagram for the bifurcations that replace the transcritical riddling bifurcation shown in the Fig. 4, in the case of a small parameter mismatch $\delta=0.998$.

another in an inverse saddle-node bifurcation. At some larger value $\varepsilon=\varepsilon_{s n}^{\prime \prime}>\varepsilon_{s n}^{\prime}$ a new saddle-node bifurcation occurs that results in the birth of two new fixed points (repelling node and saddle) below the diagonal. Subsequently, one of them (the saddle) moves away from the diagonal and the other (the repellor) approaches it. An example of the bifurcation diagram is shown in Fig. 13.

The crisis bifurcation at $\varepsilon=\varepsilon_{s n}^{\prime}$ of the absorbing area $\widetilde{I}_{D}$ can be considered as an analog of the hard riddling bifurcation in the presence of parameter mismatch. Indeed, before the bifurcation, the trajectories cannot escape from the thin absorbing area $\widetilde{I}_{D}$. Asynchronous bursts remain small and grow smoothly with the magnitude of mismatch and coupling: the maximal amplitude of the bursts is determined (approximately) by the deviation of the point $P_{0}$ from the diagonal. After the bifurcation the invariance of $\widetilde{I}_{D}$ is destroyed. The trajectories starting from $\widetilde{I}_{D}$ get access to a neighborhood of the saddle point $P$ located far away from the diagonal. Further, behavior of the trajectories is similar to that described in the Sec. III: following the unstable manifolds of $P$ they fold at $L_{1}$ or $L_{2}$ and come close to the diagonal. Then trajectories spend some (usually long) time within the former region $\widetilde{I}_{D}$ before entering a new burst to $P$; and this type of behavior will be repeated. This crisis of $\widetilde{I}_{D}$ can be considered as interior. It replaces the transcritical riddling bifurcation of the symmetrical system in the case when riddling results in a locally riddled basin.

If, at $\varepsilon=\varepsilon_{s n}^{\prime}$, the fixed point $Q$ brings with it part of the basin of an attractor $A_{u p}$ developed from $P$, or part of the basin of infinity, the crisis of $\widetilde{I}_{D}$ is exterior. In this case, after the crisis, most of the trajectories from $\widetilde{I}_{D}$ will move to the attractor $A_{u p}$ over the diagonal, or escape to infinity, so that they will never come back to $\widetilde{I}_{D}$. The exterior crisis replaces the transcritical riddling bifurcation of the symmetrical system in the case when riddling results in a globally riddled basin.

Thus, we have exposed the moment when the smoothly growing small desynchronous bursts in the system with mismatch (14) are abruptly changed into the appearance of excursions far away from the diagonal. This occurs when an inverse saddle-node bifurcation of the repellor $Q$ and the

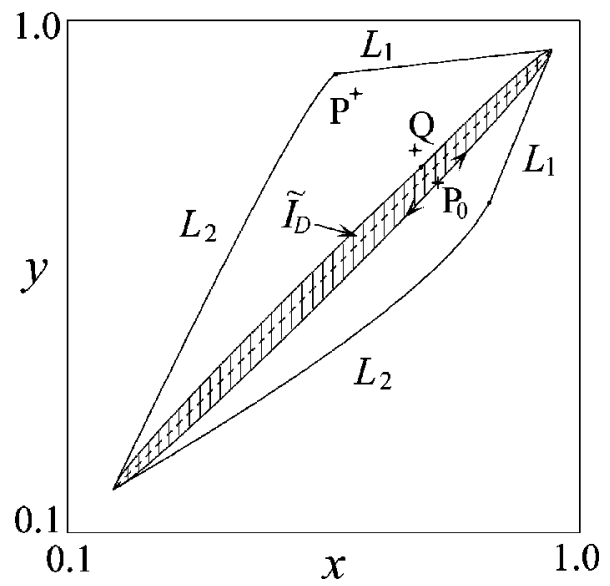

FIG. 14. Absorbing area $\widetilde{I}_{D}$ (crosshatched) replacing the synchronous state $I_{D}$ (dashed) in the case of a parameter mismatch $\delta$ $=1.002$. Parameters are $a=3.8, \varepsilon=1.55$, and $p=0.4$.

saddle fixed point $P_{0}$ destroys the thin invariant absorbing area $\widetilde{I}_{D}$.

Now we consider the case with $\delta=1.002$. At such a mismatch the saddle fixed point $P_{0}$ is placed slightly below $D$. Unstable manifolds of $P_{0}$ again bound an invariant absorbing area $\widetilde{I}_{D}$, as it is shown in Fig. 14 .

With increase of $\varepsilon$, two fixed points $P$ and $Q$ arise above the diagonal in a saddle-node bifurcation. Then, the repelling node $Q$ moves toward the diagonal. At the same time, $P_{0}$ slowly moves down and away from the diagonal, leading to an increasing size of the absorbing area $\widetilde{I}_{D}$. The bifurcation diagram is shown in Fig. 15.

With further increase of $\varepsilon$, the repelling node $Q$ enters into the absorbing area $\widetilde{I}_{D}$ and destroys it. Again, this is the moment of a crisis bifurcation of $\widetilde{I}_{D}$, which is an analog of the hard transcritical riddling bifurcation for the case of parameter mismatch. Asynchronous bursts of the trajectories abruptly grow so that the trajectories can go far away from the diagonal. In analogy with the above description, the further behavior depends on whether there is an attractor $A_{u p}$ above the diagonal, and whether an absorbing area $\mathcal{A}$ still exists. After the crisis, trajectories from the former absorbing area $\widetilde{I}_{D}$ may go to the attractor $A_{u p}$ (if it exists), or escape to

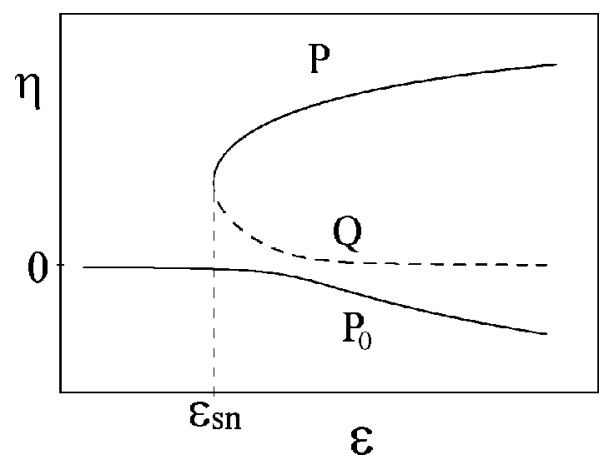

FIG. 15. Diagram of the bifurcations that replace the transcritical riddling bifurcation shown in Fig. 4 in the case of a small parameter mismatch $\delta=1.002$. 


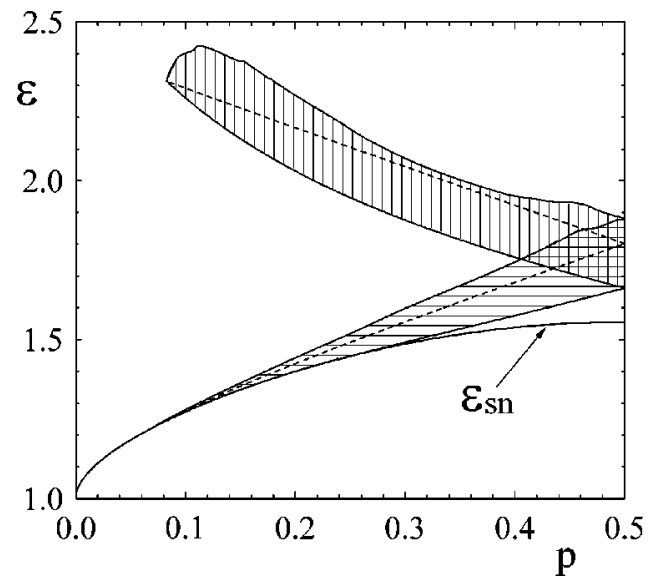

FIG. 16. Regions in the parameter $(p, \varepsilon)$ plane for the existence of the attractor $A_{u p}$ above the diagonal (horizontally hatched) and attractor $A_{d n}$ below the diagonal (vertically hatched). Curve of saddle-node bifurcation of the fixed points $P$ and $Q$ is also shown (denoted by $\varepsilon_{s n}$ ). Parameter is $a=3.8$.

infinity (if the absorbing area $\mathcal{A}$ is destroyed), or (otherwise) fill the whole area $\mathcal{A}$.

Therefore, in both the cases $\delta<1$ and $\delta>1$, the crisis bifurcation of $\widetilde{I}_{D}$ appears as a hard symmetry-breaking bifurcation of the system (14). Hard transcritical riddling and following blowout bifurcations for the case $\delta=1$ are replaced by the interior or exterior crisis of the thin invariantabsorbing region $\widetilde{I}_{D}$ in place of $I_{D}$.

\section{ATTRACTORS OUT OF THE DIAGONAL}

After the above discussion of the phenomena that arise in the presence of subpopulations of maps with slightly different parameters, let us now return to the map (3) in order to briefly discuss the bifurcations that occur for attracting states situated outside the main diagonal $D$. As described in Sec. IV, these attractors play an important role in the riddling and blowout transitions of the synchronous state $I_{D}$. At the same time they represent a two-dimensional dynamics associated with the two-cluster states (2) in the system of $N$ globally coupled maps (1).

Consider the case $\varepsilon>1$. Then, with increasing parameter $\varepsilon$ the saddle-node bifurcation occurs at some $\varepsilon=\varepsilon_{s n}$. The bifurcation results in the appearance of two fixed points $P$ and $Q$ above the diagonal (Fig. 4). For proper values of $p$ the fixed point $P$ becomes an attracting node, and later, gives rise to another attractor $A_{u p}$, which can be regular or chaotic. Finally, the attractor $A_{u p}$ is destroyed in a boundary crisis. The region of existence for the attractor $A_{u p}$ is horizontally crosshatched in Fig. 16 (see also Fig. 7).

At the same time, the repelling node $Q$ approaches the symmetrical saddle fixed point $P_{0} \in I_{D}$ and passes through it in the transcritical bifurcation (Fig. 4). $Q$ moves below the diagonal and becomes a saddle. With further increase of $\varepsilon, Q$ stabilizes in an inverse subcritical period-doubling bifurcation, and hereafter gives birth to the attractor $A_{d n}$ (below the diagonal) that may be regular or chaotic. The attractor $A_{d n}$ disappears through a boundary-crisis bifurcation. The region

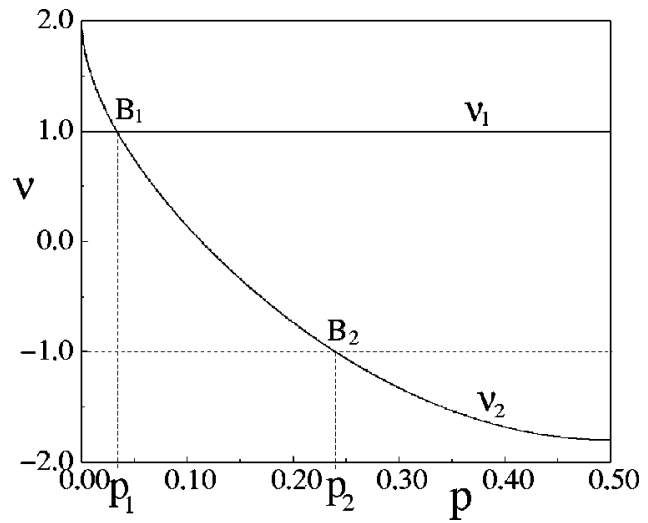

FIG. 17. Graphs of the eigenvalues $\nu_{i}, i=1,2$ of the fixed point $P=Q$ calculated for a parametric point $(p, \varepsilon)$ moving along the curve of saddle-node bifurcation $\left\{(p, \varepsilon) \mid \varepsilon=\varepsilon_{s n}(p), 0 \leqslant p \leqslant \frac{1}{2}\right\}$ (Fig. 16). Codimension-2 bifurcations occur at $p_{1} \approx 0.033$ and $p_{2} \approx 0.24$. Parameter is $a=3.8$.

of existence for the attractor $A_{d n}$ is vertically crosshatched in Fig. 16.

It was shown above that the fixed points $P$ and $Q$ are responsible for the appearance of the attractors $A_{u p}$ and $A_{d n}$ out of the diagonal for $\varepsilon>1$. Let us next study in more detail the bifurcations of $P$ and $Q$. For this, the eigenvalues $\nu_{i}(P)$ and $\nu_{i}(Q), i=1,2$ have to be examined. They are the roots $\nu_{1,2}$ of the quadratic equation

$$
\begin{aligned}
& \nu^{2}+\left(2 a(2 p-1) \varepsilon \eta^{ \pm}-\frac{\varepsilon-2}{\varepsilon-1}\right) \nu+4 a^{2}(\varepsilon-1) \eta^{ \pm} \\
& -\frac{1}{\varepsilon-1}=0
\end{aligned}
$$

Having fixed the nonlinearity parameter $a$, we move the parameter point $(p, \varepsilon)$ along the curve $\left\{(p, \varepsilon) \mid \varepsilon=\varepsilon_{s n}(p), 0\right.$ $\left.\leqslant p \leqslant \frac{1}{2}\right\}$ of the saddle-node bifurcation (see Fig. 16) where the fixed points $P$ and $Q$ coincide. In Fig. 17 both eigenvalues $\nu_{1}$ and $\nu_{2}$ of the fixed point $P=Q$ are plotted versus the parameter $p \in[0,1 / 2]$. One of the eigenvalues equals +1 whereas the other decreases monotonically from 2 to $2-a$ when $p$ varies from 0 to 0.5 .

For any $a \in(3 ; 4)$ there are two singular points $B_{1}\left(p_{1}, \varepsilon_{1}\right)$ and $B_{2}\left(p_{2}, \varepsilon_{2}\right)$ such that both eigenvalues above satisfy $\left|\nu_{i}\right|=1$. These codimension-2 bifurcation points are of distinct types: in $B_{1}$ both eigenvalues equal +1 , and in $B_{2}$ they equal +1 and -1 . In the mathematical literature, the singular point $B_{1}$ is referred to as a $1: 1$ strong resonance [41].

Let us now calculate the eigenvalues of $P$ when the parameter point $(p, \varepsilon)$ moves along the bifurcations curve of stabilization of the fixed point $P$ (lower boundary of the horizontally crosshatched region in Fig. 16). Both eigenvalues are real here, their graphs are depicted in Fig. 18 as two solid curves. Alternatively, let the parameter point $(p, \varepsilon)$ move along the bifurcation curve of destabilization of the fixed point $P$ (dashed curve within the horizontally crosshatched region in Fig. 16). In this case both eigenvalues of $P$ are 


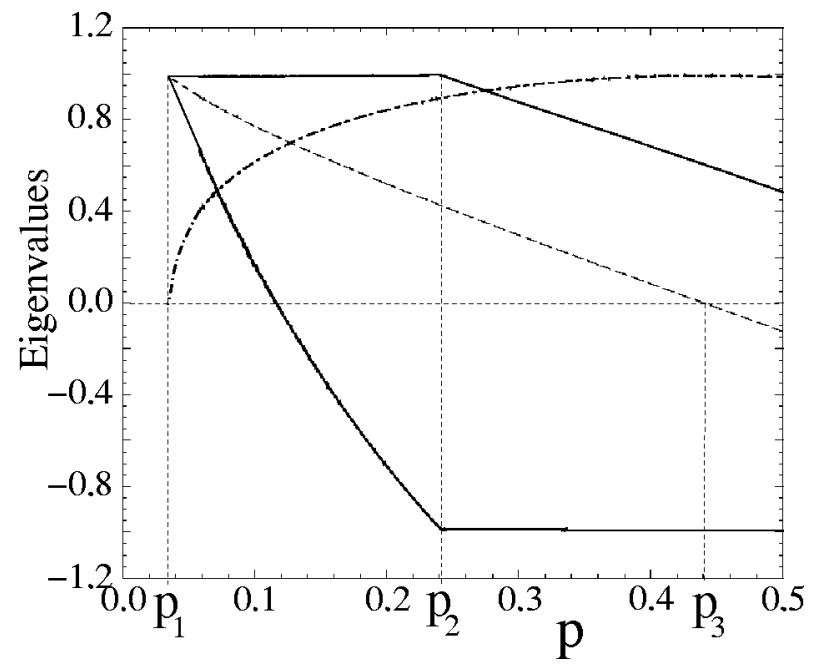

FIG. 18. Eigenvalues of the fixed point $P$ calculated when parameter point $(p, \varepsilon)$ moves along the bifurcation curves of stabilization and destabilization of $P$. A 1:4 strong resonance occurs at $p_{3} \approx 0.42$. Parameter is $a=3.8$.

complex, graphs of their real and imaginary parts are represented in Fig. 18 as dashed and dot-dashed curves, respectively.

As one can see from Fig. 18, when fixing parameter $p$ $\in\left(p_{2} ; 0.5\right]$ and increasing $\varepsilon$, the fixed point $P$ stabilizes through an inverse subcritical period-doubling bifurcation: one of its eigenvalues enters the unit circle through -1 . When $p \in\left(p_{1} ; p_{2}\right), P$ is born and becomes stable just in the moment of saddle-node bifurcation at $\varepsilon=\varepsilon_{s n}$ : the eigenvalue of $P$ enters the unit circle through +1 . When $p$ $\in\left[0 ; p_{1}\right)$ the fixed point $P$ does not stabilize at all. Inspection of Fig. 18 also shows that the fixed point $P$ loses its stability in a Hopf bifurcation, everywhere except for the point $p=p_{3}$ where the imaginary part is equal to zero and hence, a 1:4 strong resonance takes place.

An example of a chaotic attractor $A_{u p}$ developed from $P$ is shown in Fig. 19(a). With further increase of $\varepsilon$ this attractor grows in size and touches its basin boundary causing the boundary crisis. The crisis situation is shown in Fig. 19(b) (the boundary of $A_{u p}$ is created by eight arcs of critical curves $L_{k}$ ).

Let us now briefly outline the bifurcations of the fixed point $Q$ and the attractor $A_{d n}$ developed from $Q$. After the transcritical bifurcation, the saddle fixed point $Q$ stabilizes (for proper values of $p$ ) through an inverse subcritical period-doubling bifurcation (lower boundary curve of the vertically crosshatched region in Fig. 16) and becomes an attracting node. With further increase of $\varepsilon, Q$ changes from a stable node to a stable focus and then undergoes a Hopf bifurcation (dashed bifurcation curve within the vertically crosshatched region in Fig. 16) giving birth to an attractor $A_{d n}$ below the diagonal. Finally, the attractor $A_{d n}$ is destroyed in a boundary crisis (upper bifurcation curve of the vertically crosshatched region in Fig. 16).

As it can be concluded by calculating eigenvalues of $Q$, depending on $p$, there is a possibility for $Q$ to bifurcate through a 1:2 strong resonance (when both eigenvalues are
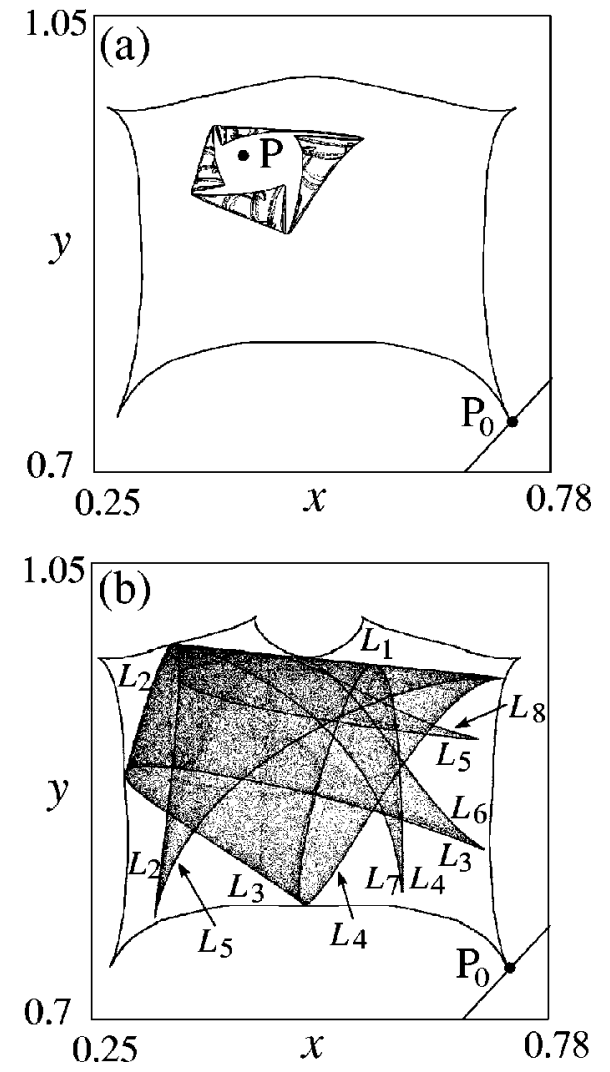

FIG. 19. Attractor $A_{u p}$ over the diagonal for the map (3) and its basin boundary. Parameters are $a=3.8, p=0.4$, (a) $\varepsilon=1.71$ (after the destruction of the closed invariant curve), (b) $\varepsilon=1.744$ (in the moment of boundary crisis bifurcation).

real and equal to -1 ) and through a 1:3 strong resonance (when the eigenvalues are complex such that their real parts are equal to -0.5 ). At $a=3.8$ these codimension-2 bifurcations take place at $p \approx 0.082$ and $p \approx 0.318$, respectively.

With further variations in $\varepsilon$, depending on $p$, different bifurcation transitions of attractors under the diagonal are observed. They will be reported in more detail elsewhere.

\section{CONCLUSION}

The transition from fully synchronized behavior to twocluster dynamics was investigated for a system of $N$ globally coupled logistic maps. If the oscillators happen to distribute themselves symmetrically between the two clusters, the transition takes place either via a transverse period-doubling or via a transverse pitchfork bifurcation. This situation was recently considered by a number of authors, and the main idea of the present work was to extend these investigations to examining the influence of an asymmetric distribution of oscillators between the two clusters, as this is the more generic as well as the physically more realistic case. We also studied the particular phenomena that can arise in the presence of two subpopulations of maps with slightly different parameters.

Our first observation was that whereas the transverse period-doubling bifurcation remains essentially unaffected by an uneven distribution of the oscillators, the nongeneric 
character of the pitchfork bifurcation causes it to be replaced by a saddle-node bifurcation in which a saddle cycle and a repelling point cycle are born off the main diagonal. This is followed by a transcritical riddling bifurcation as the repelling point causes the synchronization manifold and exchanges its transverse stability for a synchronous saddle cycle. An important consequence of this replacement of the bifurcation structure is that instead of a soft riddling transition (after a supercritical pitchfork bifurcation), a hard transition is observed. This implies that the bursts away from the synchronous state can attain finite amplitudes immediately after the riddling transition.

The transcritical riddling bifurcation itself is nongeneric, and in the presence of two subpopulations of maps with slightly different parameters, it is replaced either by two subsequent saddle-node bifurcations, in which the nearly symmetric synchronized saddle solution existing before the bifurcation is replaced by a repelling solution, or by the smooth shift of the saddle solution away from the synchronization manifold. In both cases the result is that instead of the transcritical riddling bifurcation, we observe a symmetrybreaking bifurcation in which a narrow invariant region around the symmetric chaotic state is destroyed.

Finally, we followed the bifurcations that take place after the riddling bifurcation for attractors outside the synchronization manifold. Depending on the parameters $\varepsilon$ and $p$ that control the coupling strength and the asymmetry in the distribution of oscillators, a variety of different bifurcation se- quences can be observed. Particularly interesting in this connection was the observation of several codimension-2 bifurcation points that to our knowledge has not previously been considered in detail for maps.

The concept of an absorbing area has served as one of the main tools in the above investigations. This concept derives from the theory of noninvertible two-dimensional maps with the images of the so-called critical curves playing the essential role of nonlinear restraints on the excursions of the trajectories in phase space. A somewhat similar role is played by the unstable manifolds of saddle cycles situated off the main diagonal. These concepts allowed us to characterize both the riddling and the blowout bifurcations in terms of their type as soft or hard transitions. As demonstrated by our investigations, the one-to-two cluster transition in systems of globally coupled chaotic maps depends heavily on the symmetries imposed on the problem. The symmetries associated with many cluster dynamics are extremely rich, and a detailed study of the corresponding transitions is far from trivial. We consider the discussion of one-to-two cluster transitions as only a first step in developing a more detailed understanding of globally coupled chaotic systems.

\section{ACKNOWLEDGMENTS}

Yu.M. and O.P. acknowledge support of the VW-Stiftung und DFG (Sonderforschungsbereich 555). We thank K. Kaneko for useful discussions.
[1] G. Nicolis and I. Prigogine, Self-Organization in Nonequilibrium Systems (Wiley, New York, 1977).

[2] H. Haken, Synergetics: An Introduction (Springer, Berlin, 1978).

[3] A. T. Winfree, The Geometry of Biological Time (Springer, Berlin, 1980).

[4] E. Sismondo, Science 249, 55 (1990).

[5] R. M. Santos, L. M. Rosario, A. Nadal, J. Garcia-Sancho, B. Soria, and M. Valdeomillos, Pfluegers Arch. 418, 417 (1991).

[6] S. Danф, P. G. Sørensen, and F. Hynne, Nature (London) 412, 320 (1999).

[7] P. Hadley, M. R. Beasley, and K. Wiesenfeld, Phys. Rev. B 38, 8712 (1988).

[8] K. Wiesenfeld, C. Bracikowski, G. James, and R. Roy, Phys. Rev. Lett. 65, 1749 (1990).

[9] S. H. Strogatz, C. M. Marcus, R. M. Westervelf, and R. E. Mirollo, Physica D 36, 23 (1989).

[10] Y. Kuramoto, Prog. Theor. Phys. Suppl. 79, 223 (1984); H. Sakaguchi, S. Shinomoto, and Y. Kuramoto, Prog. Theor. Phys. 77, 1005 (1987).

[11] G.B. Ermentrout and N. Kopell, SIAM (Soc. Ind. Appl. Math.) J. Math. Anal. 15, 215 (1984); N. Kopell and G. B. Ermentrout, Commun. Pure Appl. Math. 39, 623 (1986).

[12] H. Daido, Prog. Theor. Phys. 75, 1460 (1986); 77, 622 (1987); J. Stat. Phys. 60, 753 (1990).

[13] A. T. Winfree, J. Theor. Biol. 16, 15 (1967).

[14] H. Bohr, K. S. Jensen, T. Petersen, B. Rathjen, E. Mosekilde, and N.-H. Holstein-Rathlou, Parallel Comput. 12, 113 (1989).

[15] A. Pikovsky, M. G. Rosenblum, and J. Kurths, Europhys. Lett. 34, 165 (1996).

[16] M. Rosenblum, A. Pikovsky, and J. Kurths, Phys. Rev. Lett. 76, 1804 (1996).

[17] D. H. Zanette and A. S. Mikhailov, Phys. Rev. E 57, 276 (1998).

[18] J. Milnor, Commun. Math. Phys. 99, 177 (1985); 102, 517 (1985).

[19] J. C. Alexander, J. A. Yorke, Z. You, and I. Kan, Int. J. Bifurcation Chaos Appl. Sci. Eng. 2, 795 (1992).

[20] K. Kaneko, Physica D 41, 137 (1990); 75, 55 (1994); 124, 322 (1998).

[21] V. I. Sbitnev, Int. J. Bifurcation Chaos Appl. Sci. Eng. 6, 1495 (1996); K. Kaneko, Phys. Rev. Lett. 78, 2736 (1997); F. Xie and G. Hu, Phys. Rev. E 56, 1567 (1997); M. Hasler, Yu. Maistrenko, and O. Popovych, ibid. 58, 6843 (1998); V. R. Mendes, Phys. Lett. A 257, 132 (1999); Yu. Maistrenko, O. Popovych, and M. Hasler, Int. J. Bifurcation Chaos Appl. Sci. Eng. 10, 179 (2000); A. V. Taborov, Yu. L. Maistrenko, and E. Mosekilde, ibid. 10, 1051 (2000).

[22] Yu. L. Maistrenko, V. L. Maistrenko, A. Popovich, and E. Mosekilde, Phys. Rev. E 57, 2713 (1998).

[23] Yu. L. Maistrenko, V. L. Maistrenko, A. Popovych, and E. Mosekilde, Phys. Rev. E 60, 2817 (1999); Phys. Lett. A 262, 355 (1999).

[24] Yu. L. Maistrenko, V. L. Maistrenko, A. Popovich, and E. Mosekilde, Phys. Rev. Lett. 80, 1638 (1998). 
[25] C. Mira, L. Gardini, A. Barugola, and J.-C. Cathala, Chaotic Dynamics in Two-Dimensional Noninvertible Maps (World Scientific, Singapore, 1996); C. E. Frouzakis, L. Gardini, I. G. Kevrekidis, G. Millerioux, and C. Mira, Int. J. Bifurcation Chaos Appl. Sci. Eng. 7, 1167 (1997); G. Millerioux and C. Mira, ibid. 8, 2019 (1998).

[26] P. Ashwin and J. R. Terry, Physica D 142, 87 (2000).

[27] V. Astakhov, A. Shabunin, T. Kapitaniak, and V. Anishchenko, Phys. Rev. Lett. 79, 1014 (1997).

[28] V. Astakhov, M. Hasler, T. Kapitaniak, A. Shabunin, and V. Anishchenko, Phys. Rev. E 58, 5620 (1998).

[29] Y.-C. Lai and C. Grebogi, Phys. Rev. E 53, 1371 (1996); Y.-C. Lai, C. Grebogi, J. A. Yorke, and S. C. Venkataramani, Phys. Rev. Lett. 77, 55 (1996).

[30] P. Ashwin, J. Buescu, and I. Stewart, Nonlinearity 9, 703 (1996); P. Ashwin, P. J. Aston, and M. Nicol, Physica D 111, 81 (1998).

[31] S. C. Venkataramani, B. R. Hunt, and E. Ott, Phys. Rev. Lett.
77, 5361 (1996); Phys. Rev. E 54, 1346 (1996).

[32] Yu. Maistrenko, T. Kapitaniak, and P. Szuminski, Phys. Rev. E 56, 6393 (1997); T. Kapitaniak, Yu. Maistrenko, A. Stefanski, and J. Brindley, ibid. 57, R6253 (1998).

[33] B. R. Hunt and E. Ott, Phys. Rev. Lett. 76, 2254 (1996).

[34] V. I. Arnold, Theory of Ordinary Differential Equations (Nauka, Moskow, 1978), Pt. II.

[35] G. Iooss and D. D. Joseph, Elementary Stability and Bifurcation Theory (Springer, New York, 1997).

[36] J. C. Sommerer and E. Ott, Nature (London) 365, 138 (1993); E. Ott and J. C. Sommerer, Phys. Lett. A 188, 39 (1994).

[37] A. S. Pikovsky and P. Grassberger, J. Phys. A 24, 4587 (1991).

[38] P. Glendinning, Phys. Lett. A 264, 303 (1999).

[39] S. E. Newhouse, Topology 13, 9 (1974).

[40] S. V. Gonchenko, L.P. Shil'nikov, and D. V. Turaev, Chaos 6, 15 (1996).

[41] Yu. Kuznetsov, Elements of Applied Bifurcation Theory (Springer, New York, 1995). 